IZA DP No. 6806

Evolution of Teachers' Salaries in Latin America at the Turn of the 20th Century: How Much Are They (Under or Over) Paid?

Alejandra Mizala

Hugo Ñopo

August 2012 


\title{
Evolution of Teachers' Salaries in Latin America at the Turn of the 20th Century: How Much Are They (Under or Over) Paid?
}

\author{
Alejandra Mizala \\ Universidad de Chile \\ Hugo Ñopo \\ Inter-American Development Bank \\ and IZA \\ Discussion Paper No. 6806 \\ August 2012 \\ IZA \\ P.O. Box 7240 \\ 53072 Bonn \\ Germany \\ Phone: +49-228-3894-0 \\ Fax: +49-228-3894-180 \\ E-mail: iza@iza.org
}

Any opinions expressed here are those of the author(s) and not those of IZA. Research published in this series may include views on policy, but the institute itself takes no institutional policy positions.

The Institute for the Study of Labor (IZA) in Bonn is a local and virtual international research center and a place of communication between science, politics and business. IZA is an independent nonprofit organization supported by Deutsche Post Foundation. The center is associated with the University of Bonn and offers a stimulating research environment through its international network, workshops and conferences, data service, project support, research visits and doctoral program. IZA engages in (i) original and internationally competitive research in all fields of labor economics, (ii) development of policy concepts, and (iii) dissemination of research results and concepts to the interested public.

IZA Discussion Papers often represent preliminary work and are circulated to encourage discussion. Citation of such a paper should account for its provisional character. A revised version may be available directly from the author. 


\begin{abstract}
Evolution of Teachers' Salaries in Latin America at the Turn of the 20th Century: How Much Are They (Under or Over) Paid?*

How much are teachers paid in comparison to those in other professions in Latin America? How have these differences evolved at the turn of the $20^{\text {th }}$ century? This paper reports the evolution, between circa 1997 and circa 2007, of teachers' salaries vis-à-vis workers in other professional and technical occupations for thirteen Latin-American countries. After controlling the earnings differentials by observable characteristics linked to productivity it is found that the hourly earnings gap, although substantial, decreased throughout the decade. This has been the case for earnings gaps at the main and secondary jobs, and also for those measured in terms of monthly and yearly earnings. Nonetheless, behind the region averages there is an important cross-country heterogeneity.
\end{abstract}

JEL Classification: J31, J44, J8, O54

Keywords: wage differentials, professional labor markets, national and international labor standards, Latin America, Caribbean

Corresponding author:

Hugo Ñopo

Inter-American Development Bank

Colombia Country Office

Carrera 7ma 71-21, Torre B, Piso 19

Bogotá

Colombia

E-mail: hugon@iadb.org

\footnotetext{
*Felipe Balcazar provided very valuable research assistance. We thank participants at seminars at the Economics Department at University of Chile, SECHI and LACEA Annual Meetings for comments and suggestions. Any mistake within the paper is our own and the findings herein do not necessarily represent the views of the Inter-American Development Bank or its Board of Directors. Mizala thanks financial support from PIA-CONICYT Project CIE-05 and Fondecyt project 1100308.
} 


\section{Introduction}

School teachers' salaries are often perceived to be lower than those of other professionals; this perception is especially strong among teachers. This affects the teachers' motivation to educate (OECD 2009; Figlio and Kenny 2006; Ortega 2010, Player 2009, Heutel 2009, Loeb and Page, 2000); causes good teachers to leave the profession (Imazeki 2005; Harris and Adams, 2007; Scafidi et al., 2007); and good students to avoid choosing an education major in college. These in turn would produce negative effects in students' learning. To improve the quality of education it is essential to pay special attention to teachers and to implement policies to attract, motivate and retain the most talented individuals in the profession.

A series of studies have analyzed teachers' salaries, with mixed results regarding their relative under or over-payment in the labor markets. In fact, the available empirical evidence shows that the sign and the magnitude of the conditional wage differential between teachers and other workers crucially depends on the definition of the comparison group, even when differences in observable characteristics are accounted for, both in Latin America ${ }^{1}$ and out of the region ${ }^{2}$.

More recently Mizala and Ñopo (2011), analyzing representative samples of nine Latin American countries circa 2007, found that teachers' underpayment is stronger than what has been previously reported in the literature for Latin America. Teachers' underpayment with respect to other professionals and technicians was found to be more pronounced among males, older workers, household heads, part-timers, formal workers, those who work in the private sector, and (mostly) among those with complete tertiary education. Exploring the role of job schedules and job tenure (which are claimed to be more flexible and longer, respectively, for teachers) as compensating differentials, it was found that even after accounting for them the conditional earnings gap prevails. This paper builds up on these results, expanding them in three important dimensions: (i) exploring the evolution of those conditional earning gaps between circa 1997 and circa 2007, (ii) expanding the number of countries to thirteen, improving on its representativeness, and (iii) exploring deeper into the role of individuals' unobservable characteristics by using information from their main and second jobs.

The question of earnings differentials conditioning on observable characteristics is assessed with a non-parametric matching approach developed in Ñopo (2008). The results depict a picture in which teachers, still today, are underpaid vis-à-vis other professionals and technicians. We found, however, that preschool and elementary teachers' earnings (vis-à-vis those of other professionals and technicians) improved during the decade, especially for the young, females, part-time workers

\footnotetext{
${ }^{1}$ Psacharopoulos et al. (1996), Liang (1999), and Hernani-Limarino (2005) analyze several Latin American countries. Saavedra (2004) examines Perú, Mizala and Romaguera (2005) Chile, Lopez-Acevedo (2004) Mexico, Rivas and Lavarreda (2008) Guatemala, Herreros et al. (2003) Argentina, Piras and Savedoff (1998) and Urquiola et al. (2000) Bolivia.

2 Taylor (2008), Allegretto et al (2008), Podgursky and Tongrut (2006), Harris and Adams (2005), Stoddard (2005) for United States, Asadullah (2006) for Bangladesh; Komenan and Grootaert (1990) for Cote D' Ivoire; Zymelman and DeStephano (1989) for Sub-Saharan African countries.
} 
and those holding only one job. Also, teachers more frequently report having more than one job compared to other professionals and technicians. We analyze the decision of having a second job, as well the earnings gaps at main and second jobs between teachers and non-teachers (restricting our comparison to those who have a second job). The gap in the second job prevails but is smaller than in the main job, and it also decreased throughout the decade.

The rest of the paper proceeds as follows. In the next section we introduce the data sources, the approach to harmonize them across countries and some descriptive statistics comparing teachers with other professionals and technicians. In section three we present the main empirical analysis of earnings gaps decompositions (analyzing its evolution during the 10-year period), exploring the role of the two extra amenities: (i) shorter and flexible job schedules (with the consequent possibility of holding an extra job), and (ii) more job stability. In the fourth section we summarize and conclude.

\section{The Data}

The data comes from nationally representative household and labor surveys of thirteen LatinAmerican countries circa 1997 and circa 2007. Table 1 reports the specifics of each data source: the country, the survey name, the year and the number of observations for the whole sample of workers ("Full Set") and the subsamples of workers that will be compared ("Pre-School and Elementary Teachers", "High School Teachers" and "Other Professionals and Technicians"). The expansion factors of each data set are used such that the relative size of each sample proportionally corresponds to the working population of each country. Table A1 in the Appendix shows the occupational categories that we used in each country to identify teachers and other professionals and technicians. University teachers and those with particular specialties (e.g., teachers for students with special needs, language instructors, sports instructors, driving instructors, and dance or art instructors) are not considered for the analysis. They are neither part of the teachers' nor of the non-teachers' population. Then, when we refer here to teachers or school teachers, we will be referring to both high school teachers and pre-school and elementary teachers.

The sample of interest (school teachers and other professionals and technicians) represents $10.2 \%$ and $14.4 \%$ of the working sample circa 1997 and circa 2007 respectively. Those who declare being teachers stand for $3.5 \%$ and $3.1 \%$, and the other professionals and technicians stand for $6.6 \%$ and $11.3 \%$ of the working sample for each period, respectively. Outliers for income at the main occupation were dropped from the data set. This comprised $1 \%$ of the working sample for both periods under analysis $(0.3 \%$ and $0.6 \%$ percent of the school teachers, and $6 \%$ and $5 \%$ percent of the other professionals and technicians for each period, respectively).

Table 1 shows that the proportion of teachers ranges from $2 \%$ to $5 \%$ in both periods and it slightly decreased for many countries: Bolivia, Brazil, Chile, El Salvador, Panama, Paraguay and Peru. On the other hand, the population of other professionals and technicians increased for all countries during the period. 
Table 1. Data Sources and Sample Sizes, by Group

\begin{tabular}{|c|c|c|c|c|c|c|c|c|c|c|}
\hline \multirow{3}{*}{ Country } & \multicolumn{10}{|c|}{ Other Professionals and Technicians/Teachers (non tertiary) Working Populations ${ }^{\star}$} \\
\hline & \multirow{2}{*}{ Name Of The Survey } & \multirow{2}{*}{ Year } & \multicolumn{2}{|c|}{ Full Set } & \multicolumn{2}{|c|}{$\begin{array}{l}\text { Pre-School and } \\
\text { Elementary Teachers }\end{array}$} & \multicolumn{2}{|c|}{ High School Teachers } & \multicolumn{2}{|c|}{$\begin{array}{l}\text { Other Professionals } \\
\text { and Technicians }\end{array}$} \\
\hline & & & $\begin{array}{l}\text { Number of } \\
\text { observations }\end{array}$ & $\begin{array}{c}\text { Expanded } \\
\text { observations }\end{array}$ & $\begin{array}{c}\text { Number of } \\
\text { observations }\end{array}$ & $\begin{array}{c}\text { Expanded } \\
\text { observations }\end{array}$ & $\begin{array}{l}\text { Number of } \\
\text { observations }\end{array}$ & $\begin{array}{c}\text { Expanded } \\
\text { observations }\end{array}$ & $\begin{array}{c}\text { Number of } \\
\text { observations }\end{array}$ & $\begin{array}{c}\text { Expanded } \\
\text { observations }\end{array}$ \\
\hline \multirow{2}{*}{ Bolivia } & \multirow{2}{*}{ Encuesta Nacional de Empleo (ENE or EE) } & 1997 & 10288 & 2218471 & 350 & 69377 & 197 & 41673 & 708 & 158542 \\
\hline & & 2009 & 8537 & 1478942 & 254 & 36549 & 181 & 25730 & 1360 & 206769 \\
\hline \multirow{2}{*}{ Brazil } & \multirow{2}{*}{ Pesquisa Nacional por Amostra de Domicilio (PNAD) } & 1995 & 110093 & 49700000 & 3406 & 1546106 & 719 & 313631 & 6217 & 2715156 \\
\hline & & 2009 & 162632 & 78400000 & 3976 & 1918232 & 1150 & 542706 & 18352 & 9006210 \\
\hline \multirow{2}{*}{ Chile } & \multirow{2}{*}{ Encuesta de Caracterizacion Socioeconomica Nacional (CASEN) } & 1998 & 61492 & 4966500 & 1388 & 123222 & 365 & 40524 & 4524 & 579730 \\
\hline & & 2009 & 82904 & 6021472 & 1535 & 113719 & 278 & 31928 & 8324 & 1003972 \\
\hline \multirow{2}{*}{ Costa Rica } & \multirow{2}{*}{ Encuesta de Hogares de Propósitos Múltiples (EHPM) } & 1995 & 12199 & 966662 & 218 & 16900 & 81 & 6541 & 720 & 68506 \\
\hline & & 2009 & 18107 & 1797512 & 345 & 34639 & 211 & 19625 & 3047 & 366669 \\
\hline \multirow{2}{*}{ Dominican Republic } & \multirow{2}{*}{ Encuesta Nacional de Fuerza de Trabajo (ENFT) } & 2000 & 8078 & 3096833 & 159 & 62525 & 29 & 10793 & 828 & 295452 \\
\hline & & 2008 & 10810 & 3479268 & 252 & 80158 & 64 & 19633 & 757 & 356222 \\
\hline \multirow{2}{*}{ Ecuador } & \multirow{2}{*}{ Encuesta de Empleo, Desempleo y Subempleo (ENEMDU) } & 2000 & 9374 & 1967617 & 257 & 46650 & 38 & 4720 & 441 & 92618 \\
\hline & & 2006 & 21694 & 5219747 & 529 & 117720 & 255 & 56533 & 1372 & 369388 \\
\hline \multirow{2}{*}{ El Salvador } & \multirow{2}{*}{ Encuesta de Hogares de Propositos Multiples (EHPM) } & 1995 & 10950 & 1553995 & 265 & 33192 & 25 & 3672 & 691 & 110980 \\
\hline & & 2009 & 24299 & 1961864 & 518 & 41415 & 54 & 4758 & 1733 & 198244 \\
\hline \multirow{2}{*}{ Honduras } & \multirow{2}{*}{ Encuesta Permanente de Hogares de Propósitos Múltiples (EPHPM) } & 1995 & 9005 & 1539817 & 232 & 36542 & 78 & 11974 & 536 & 70158 \\
\hline & & 2007 & 26588 & 1936852 & 719 & 53219 & 211 & 15042 & 2755 & 184566 \\
\hline \multirow{2}{*}{ Nicaragua } & \multirow{2}{*}{ Encuesta Nacional de Hogares sobre medicion de Niveles de Vida (EMNV) } & 1998 & 5739 & 1078232 & 181 & 31456 & 27 & 4920 & 324 & 71975 \\
\hline & & 2005 & 11023 & 1652223 & 377 & 48401 & 64 & 9292 & 578 & 115217 \\
\hline \multirow{2}{*}{ Panama } & \multirow{2}{*}{ Encuesta de Hogares (EH) } & 1995 & 11318 & 722732 & 316 & 17680 & 207 & 13360 & 924 & 65954 \\
\hline & & 2007 & 18843 & 1269338 & 395 & 24953 & 220 & 14764 & 1638 & 126569 \\
\hline \multirow{2}{*}{ Paraguay } & Encuesta de Hogares por Muestra (Mano de obra) & 1996 & 4452 & 1163769 & 83 & 22291 & 48 & 11779 & 264 & 68067 \\
\hline & Encuesta Permanente de Hogares (EPH) & 2006 & 6302 & 1692845 & 129 & 26241 & 51 & 10119 & 441 & 126717 \\
\hline Pery & Encuesta Nacional de Hogares (ENAHO) & 1997 & 10036 & 8506517 & 247 & 153138 & 180 & 141606 & 774 & 832518 \\
\hline relu & 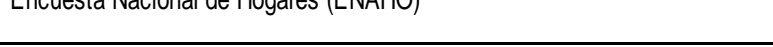 & 2009 & 33905 & 11600000 & 670 & 227592 & 388 & 130361 & 2897 & 1148469 \\
\hline Uruauav & Fncuesta Continua de Hocares (FCH) & 1998 & 21202 & 979846 & 335 & 15388 & 257 & 12233 & 1710 & 81865 \\
\hline (linguay & 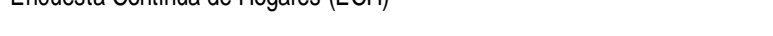 & 2007 & 25295 & 530153 & 592 & 12238 & 418 & 9023 & 2810 & 60406 \\
\hline
\end{tabular}

* Source: Authors' compilations from household surveys.

Note: Working populations in each country are identified as those earning a salary in the main occupation. 
Table 2 shows the descriptive statistics for observable characteristics in all countries' data sets for the two periods of analysis. Teaching is a predominantly female occupation as approximately nine out of ten pre-school and elementary teachers are women, and six out of ten high school teachers are so. On the other hand, the proportion of males among other professionals and technicians is roughly more than $50 \%$. Such compositions remain almost unchanged over the decade. The working population aged during the period. Workers above 45 years old increased their share in all comparison groups but this has been more pronounced in the case of teachers.

Pre-school and elementary school teachers became more professionalized during the decade as the percentage of those with tertiary complete education jumped from $13 \%$ to $19 \%$. This did not happen among neither high-school teachers nor other professionals and technicians. In all comparison groups the share of workers with secondary education increased while the share of those with lower education decreased. Teachers' educational achievement surpasses that of other professionals and technicians and this has not changed during the decade.

In both periods around $50 \%$ of pre-school and elementary teachers report living with children (12 years old or younger), while around $45 \%$ of high school teachers and non-teachers do so. The trend during the decade, however, shows a decrease in fertility in all workers' households. Also teachers, particularly high school teachers, report living with elder people (65 years or older) in a higher proportion than the other groups. Household headship has been less prevalent among teachers than among other professionals and technicians, but increased for the former during the decade. Similarly, presence of other household member generating labor income, another proxy for financial responsibility, reveals the shortening of differences between teachers and other professionals and technicians. The proportion of teachers working part-time (30 hours or less per week), although has declined, is almost threefold than that of other professionals and technicians. Even more interesting, not only a higher proportion of teachers have a secondary job, but also such share increased over the decade. During the same period such share decreased for other professionals and technicians. 
Table 2. Descriptive Statistics, by Group

\begin{tabular}{|c|c|c|c|c|c|c|}
\hline & \multicolumn{2}{|c|}{$\begin{array}{c}\text { Pre-School and } \\
\text { Elementary Teachers }\end{array}$} & \multicolumn{2}{|c|}{ High School Teachers } & \multicolumn{2}{|c|}{$\begin{array}{c}\text { Other Professionals and } \\
\text { Technicians }\end{array}$} \\
\hline & Circa 1997 & Circa 2007 & Circa 1997 & Circa 2007 & Circa 1997 & Circa 2007 \\
\hline \multicolumn{7}{|l|}{ Personal Characteristics } \\
\hline Men (gender) & $12.2 \%$ & $13.1 \%$ & $39.1 \%$ & $38.6 \%$ & $55.8 \%$ & $57.2 \%$ \\
\hline \multicolumn{7}{|l|}{ Age groups } \\
\hline 24 and under & $17.5 \%$ & $10.3 \%$ & $10.9 \%$ & $7.9 \%$ & $12.8 \%$ & $15.1 \%$ \\
\hline 25 to 34 & $37.6 \%$ & $31.4 \%$ & $31.6 \%$ & $25.7 \%$ & $35.6 \%$ & $33.8 \%$ \\
\hline 35 to 44 & $28.7 \%$ & $31.5 \%$ & $33.4 \%$ & $30.7 \%$ & $30.2 \%$ & $24.1 \%$ \\
\hline 45 to 54 & $13.7 \%$ & $19.8 \%$ & $20.2 \%$ & $23.9 \%$ & $14.9 \%$ & $18.0 \%$ \\
\hline 54 and over & $2.5 \%$ & $7.0 \%$ & $3.9 \%$ & $11.8 \%$ & $6.4 \%$ & $9.0 \%$ \\
\hline \multicolumn{7}{|l|}{ Education level } \\
\hline None or primary incomplete & $5.1 \%$ & $0.2 \%$ & $3.2 \%$ & $0.0 \%$ & $7.5 \%$ & $4.5 \%$ \\
\hline Primary complete or secondary incomplete & $7.5 \%$ & $3.0 \%$ & $2.8 \%$ & $1.1 \%$ & $11.2 \%$ & $8.3 \%$ \\
\hline Secondary complete or tertiary incomplete & $74.2 \%$ & $77.9 \%$ & $55.1 \%$ & $65.5 \%$ & $57.0 \%$ & $68.6 \%$ \\
\hline Tertiary complete & $13.2 \%$ & $18.9 \%$ & $38.9 \%$ & $33.5 \%$ & $24.2 \%$ & $18.7 \%$ \\
\hline Presence of children ( $\leq 12$ years) in the household & $58.2 \%$ & $48.4 \%$ & $50.8 \%$ & $40.9 \%$ & $52.4 \%$ & $39.8 \%$ \\
\hline Presence of elder ( $\geq 65$ years) in the household & $13.0 \%$ & $14.5 \%$ & $16.9 \%$ & $16.9 \%$ & $14.1 \%$ & $13.9 \%$ \\
\hline Head of the Household & $19.5 \%$ & $30.2 \%$ & $39.0 \%$ & $43.1 \%$ & $48.7 \%$ & $46.7 \%$ \\
\hline Presence of other household member with labor income & $79.2 \%$ & $77.1 \%$ & $74.4 \%$ & $73.8 \%$ & $69.7 \%$ & $72.0 \%$ \\
\hline \multicolumn{7}{|l|}{ Labor Characteristics } \\
\hline Part time workers ( $\leq 30$ hours) & $62.5 \%$ & $55.6 \%$ & $55.2 \%$ & $48.7 \%$ & $19.8 \%$ & $19.4 \%$ \\
\hline More than one job & $15.9 \%$ & $18.8 \%$ & $23.7 \%$ & $27.9 \%$ & $13.1 \%$ & $10.7 \%$ \\
\hline
\end{tabular}

* Source: Authors' calculations from household surveys.

Table 3 shows the relative earnings at the main job of the groups being compared by observable characteristics. Earnings are computed as hourly earnings, measured in terms of purchasing power parity (PPP, US\$, 2000). Hourly earnings for each individual are computed dividing the monthly income by 4.3 times the number of hours worked in a week. ${ }^{3}$ Average school teachers' hourly earnings circa 1997 have been set equal to 100 for each country (i.e., the average hourly earnings of both, pre-school and elementary teachers and high school teachers altogether).

\footnotetext{
${ }^{3}$ The monthly income corresponds to the monthly earnings received from the main occupation in the month previous to the survey. The job schedule is captured with survey questions of the type, for example: "¿Quantas horas trabalhava normalmente por semana nesse trabalho? ¿Cuántas horas trabaja efectivamente en su empleo o actividad principal? Señale horas semanales, ¿cuántas horas efectivas al día trabajó la semana pasada? ¿Cuántas horas trabajó la semana pasada en la ocupación principal? El mes pasado, ¿cuántas horas a la semana trabajó en este negocio o empresa? ¿Cuántas horas por semana trabaja regularmente como...?; ¿Cuántas horas, días y en qué jornada trabajo efectivamente la semana anterior?". So, it can be inferred that teachers are referring not only to their effective class time but to their whole job schedule (including preparation, grading, meeting times and the like).
} 
It is shown in the table that, on average, for both periods pre-school and elementary teachers earn less than high school teachers; these in turn earn less than other professionals and technicians circa 1997 and slightly more circa 2007. However, it should be noted that during the period, preschool and elementary teachers' earnings increased during the period of analysis while that of high-school teachers slightly dropped. The most notorious change in earnings can be seen among other professional and technicians. On average it dropped almost $25 \%$ in real terms which corresponds to almost 50\% of average teachers' earnings circa 1997 . This suggests, by now, that the gap closed due to a decrease in other professionals and technicians real acquisitive capacity.

Regarding differences according to the observable characteristics of the populations the typical patterns arise. Men earn more than women, especially in the case of other professionals and technicians. Earnings increase along a worker's life span, as well as with higher educational attainment. People that live with kids, live with elder people, are not household heads and live with another wage earner tend to earn less that those who don't or are not. These differences tend to be more pronounced among other professional and technicians than among teachers. Additionally, part-time workers and those who report having more than one job earn more than those who don't. The difference between workers who have a second job and those who don't slightly decreased during the decade, while the difference between those who are part-time workers and those who are not markedly increased. 
Table 3. Relative Hourly Earnings at the Main Job, by Group

\begin{tabular}{|c|c|c|c|c|c|c|}
\hline \multicolumn{7}{|c|}{ Relative Hourly Earnings (Base: Average School Teacher Earnings circa 1997 in each Country=100) } \\
\hline & \multicolumn{2}{|c|}{$\begin{array}{c}\text { Pre-School and } \\
\text { Elementary Teachers }\end{array}$} & \multicolumn{2}{|c|}{ High School Teachers } & \multicolumn{2}{|c|}{$\begin{array}{c}\text { Other Professionals and } \\
\text { Technicians }\end{array}$} \\
\hline & Circa 1997 & Circa 2007 & Circa 1997 & Circa 2007 & Circa 1997 & Circa 207 \\
\hline Average Hourly Earninngs & 90.3 & 99.7 & 134.3 & 128.7 & 163.0 & 123.0 \\
\hline \multicolumn{7}{|l|}{ Personal Characteristics } \\
\hline \multicolumn{7}{|l|}{ Men } \\
\hline No & 89.3 & 97.6 & 132.4 & 127.8 & 141.3 & 112.8 \\
\hline Yes & 97.5 & 113.3 & 137.2 & 130.1 & 180.2 & 130.6 \\
\hline \multicolumn{7}{|l|}{ Age groups } \\
\hline 24 and under & 59.6 & 64.5 & 112.0 & 84.5 & 90.3 & 70.5 \\
\hline 25 to 34 & 83.5 & 88.1 & 123.0 & 112.7 & 153.2 & 114.1 \\
\hline 35 to 44 & 103.8 & 103.7 & 140.7 & 130.3 & 183.1 & 135.1 \\
\hline 45 to 54 & 115.0 & 118.4 & 148.4 & 140.0 & 195.1 & 150.7 \\
\hline 54 and over & 116.7 & 132.2 & 160.0 & 166.0 & 193.1 & 156.4 \\
\hline \multicolumn{7}{|l|}{ Education level } \\
\hline None or primary incomplete & 32.3 & 49.5 & 81.2 & 29.9 & 78.6 & 62.4 \\
\hline Primary complete or secondary incomplete & 45.5 & 82.8 & 111.4 & 109.4 & 93.5 & 76.3 \\
\hline Secondary complete or tertiary incomplete & 97.1 & 96.9 & 145.1 & 123.1 & 174.8 & 119.1 \\
\hline Tertiary complete & 99.8 & 114.3 & 125.0 & 140.3 & 193.6 & 172.5 \\
\hline \multicolumn{7}{|l|}{ Presence of children ( $\leq 12$ years) in the household } \\
\hline No & 96.0 & 101.5 & 137.0 & 129.7 & 170.3 & 125.1 \\
\hline Yes & 86.2 & 97.7 & 131.6 & 127.3 & 156.3 & 119.7 \\
\hline \multicolumn{7}{|l|}{ Presence of elder ( $\geq 65$ years) in the household } \\
\hline No & 90.7 & 99.3 & 135.9 & 128.1 & 164.4 & 124.0 \\
\hline Yes & 87.2 & 101.6 & 126.4 & 131.8 & 154.5 & 116.7 \\
\hline \multicolumn{7}{|l|}{ Head of the household } \\
\hline No & 87.3 & 94.4 & 127.9 & 123.1 & 134.8 & 107.0 \\
\hline Yes & 102.4 & 111.9 & 144.2 & 136.2 & 192.7 & 141.1 \\
\hline \multicolumn{7}{|c|}{ Presence of other household member with labor income } \\
\hline No & 90.2 & 100.3 & 129.2 & 129.4 & 171.5 & 128.0 \\
\hline Yes & 90.3 & 99.5 & 136.0 & 128.5 & 159.3 & 121.0 \\
\hline \multicolumn{7}{|l|}{ Labor Characteristics } \\
\hline \multicolumn{7}{|l|}{ Part time } \\
\hline No & 84.8 & 87.5 & 121.3 & 110.3 & 154.3 & 118.5 \\
\hline Yes & 93.5 & 109.3 & 144.8 & 148.1 & 197.8 & 141.6 \\
\hline \multicolumn{7}{|l|}{ More than one job } \\
\hline No & 87.1 & 97.2 & 132.0 & 125.2 & 158.1 & 120.0 \\
\hline Yes & 107.1 & 110.2 & 141.5 & 137.9 & 195.1 & 148.2 \\
\hline
\end{tabular}

* Source: Authors' calculations from household surveys

At the aggregate, on average, other professionals and technicians earn around $115 \%$ and $43 \%$ more than pre-school and elementary teachers, circa 1997 and circa 2007 respectively. Also, they earn 42\% more than high school teachers circa 1997, and 5\% less circa 2007 (although this last difference is not significant at conventional levels). These statistics, however, are merely referential. They compare teachers with professionals and technicians that might substantially differ in terms of observable characteristics. As shown above in this section, teachers and other 
professionals and technicians differ regarding their human capital, job characteristics and sociodemographic composition. Then, it is appropriate to think that these differences in observable characteristics play a role explaining the earnings differentials. Hence, controlling the earnings gap by observable characteristics becomes necessary for a better estimation of the underlying earnings gap. The next section presents computations of the earnings gaps between teachers (preschool and primary, and secondary) and other professionals and technicians after matching individuals according to their observable characteristics.

\section{Earnings Changes at the turn of the $20^{\text {th }}$ Century for teachers vis-à-vis other professionals and technicians}

The extent to which the earnings differentials can be attributed to differences in observable characteristics is explored next. This is done using matching comparisons such that each teacher is paired with a professional or technician with the same observable characteristics (for methodological details see Ñopo, 2008). The characteristics are gender, age, education, presence of kids (12 or younger) in the household, presence of elders (65 or older) in the household, whether the workers is or not household head, presence of other wage earners in the household, whether the individual has a part-time work, and whether the individual holds a secondary job (all together will be referred as the "full set" of observable characteristics). These variables are sequentially added as matching variables and the results are reported in Figure 1 . The decompositions are sequentially calculated for (i) pre-school and elementary teachers and (ii) high school teachers, for the first (circa 1997) and second (circa 2007) periods, vis-à-vis other professionals and technicians.

\subsection{Evolution of Average Earnings Gaps (controlling for observable characteristics)}

Figure 1 shows the drop in earnings gaps between the teaching groups and their comparing group of other professionals and technicians for the period under analysis (the panel "a" of the figure is for pre-school and elementary teachers and the panel " $b$ " for high school teachers). All the earnings gaps are measured in terms of percentages of the average earnings of the teaching group that takes part on the comparison. The first pair of boxes, at the left, shows the gap that remains after matching teachers and non-teachers on gender only. That is, each male teacher is compared to a male professional or technician and each female teacher to a female professional or technician. Moving to the right each pair of boxes shows the gaps that remain after adding a matching variable. In this way, the last pair of boxes show the earnings gaps between teachers and other professionals and technicians that have the same observable characteristics on nine

variables (gender, age, education, presence of children at home, presence of elders at home, an indicator for being a household head, an indicator for the presence of other income earner at home, part-time work and an indicator for holding more than one job). That is, when moving two 
boxes to the right on Figure 1 the comparison gets restricted to individuals with the same observable characteristics, adding one characteristic at a time.

The figure shows a drop in earnings gaps that is more pronounced for pre-school and elementary teachers than for high school teachers. For the latter the drop in earnings gaps vis-à-vis other professionals and technicians is not statistically significant for almost all sets of matching characteristics.

Table 4 shows the same earnings gaps by country. Similarly to Figure 1, each pair of columns of the table corresponds to the earnings gap that remains after matching on a set of observable characteristics. Within each pair of columns, the first column corresponds to circa 1997 results and the second, circa 2007 results. The first pair of columns corresponds to the original earnings gap (the one that is measured with no matching at all). Moving to the right, each pair of columns add a matching variable such that in the last column of the table, teachers and other professionals and technicians being compared have the same observable characteristics on the nine variables.

Although some countries present negative original earnings gaps, unexplained earnings gaps after controlling for the full set of observable characteristics are either positive or statistically zero. Looking at each country separately it can be seen that the original earnings gap between preschool and elementary teachers vis-à-vis other professionals and technicians decreased in most countries of the region but it did specially for Bolivia, Brazil and Dominican Republic. The only countries where such gap increased were Costa Rica and Ecuador. The gap regarding high school teachers markedly decreased for Bolivia, Brazil and Uruguay; the gap increased for Paraguay, Nicaragua and El Salvador. All in all, the original and unexplained earnings gaps dropped for both specifications of regions' average and for both pairs in comparison.

Figure 1. Confidence Intervals for the Unexplained Earnings Gap Controlling by Observable Characteristics a. Pre-School and Elementary School Teachers versus Other Professionals and Technicians

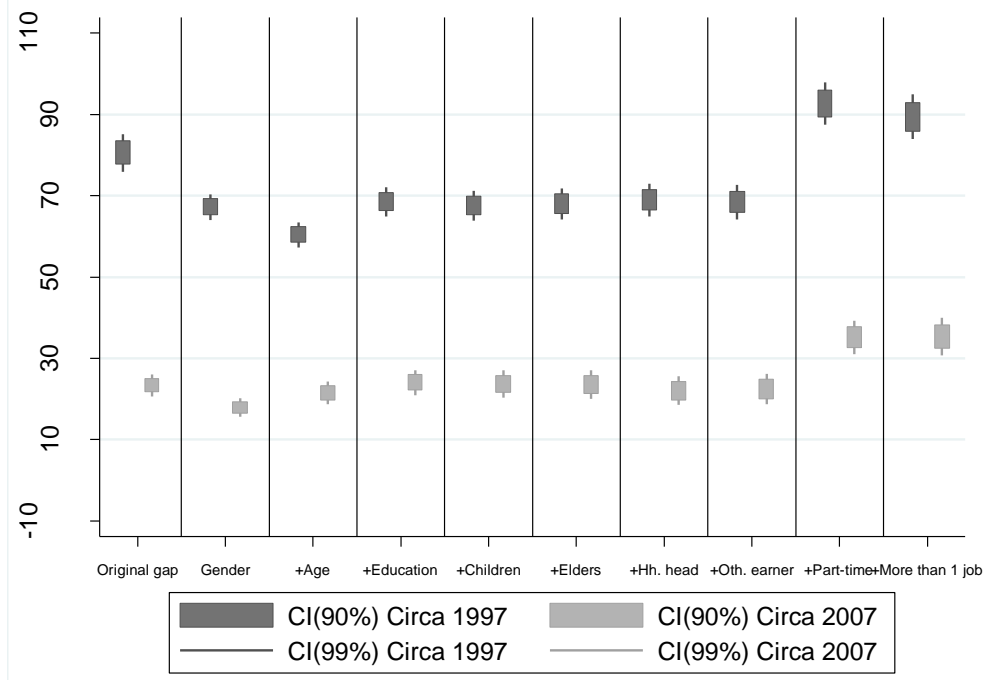

b. High School Teachers versus Other Professionals and Technicians 


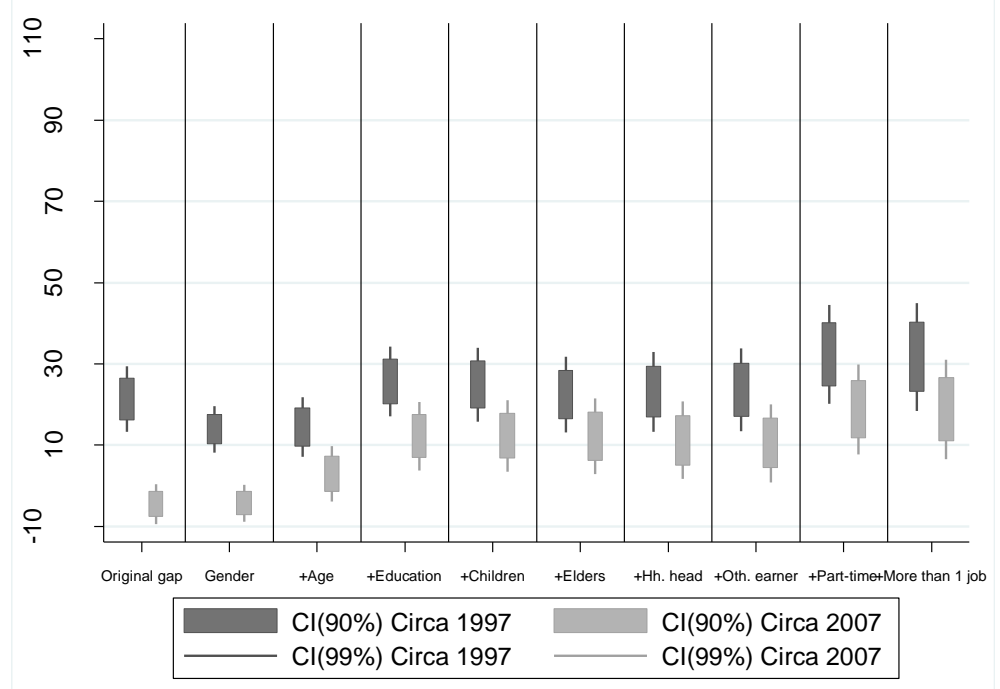

Source: Authors' calculations based on household surveys

Note: Boxes show 90 percent confidence intervals for unexplained earnings; whiskers show 99 percent confidence intervals. 
Table 4

Unexplained Earnings Gap by Country Controlling by Observable Characteristics

\begin{tabular}{|c|c|c|c|c|c|c|c|c|c|c|c|c|c|c|c|c|c|c|c|c|}
\hline \multicolumn{21}{|c|}{ Pre-School and Elementary Teachers vis-à-vis Other Professionals and Technicians } \\
\hline \multirow[t]{2}{*}{ Country } & \multicolumn{2}{|c|}{ Original gap } & \multicolumn{2}{|c|}{ +Gender } & \multicolumn{2}{|c|}{+ Age } & \multicolumn{2}{|c|}{ + Education } & \multicolumn{2}{|c|}{$\begin{array}{c}\text { Children } \\
\text { living in the } \\
\text { household } \\
\end{array}$} & \multicolumn{2}{|c|}{$\begin{array}{c}\text { + Elders living } \\
\text { in the } \\
\text { household }\end{array}$} & \multicolumn{2}{|c|}{$\begin{array}{c}+ \text { Household } \\
\text { head }\end{array}$} & \multicolumn{2}{|c|}{$\begin{array}{l}+ \text { Another wage } \\
\text { earner living in } \\
\text { the household }\end{array}$} & \multicolumn{2}{|c|}{$\begin{array}{c}\text { + Part-time } \\
\text { Work }\end{array}$} & \multicolumn{2}{|c|}{$\begin{array}{c}\text { + Has more than } \\
\text { one job }\end{array}$} \\
\hline & C-97 & C-07 & C-97 & C-07 & C-97 & C-07 & C-97 & C-07 & C-97 & C-07 & C-97 & C-07 & C-97 & C-07 & C-97 & C-07 & C-97 & C-07 & C-97 & C-07 \\
\hline Bolivia & $81.2 \%$ & $-20.4 \%$ & $\begin{array}{l}70.9 \% \\
(0.05) \\
\end{array}$ & $\begin{array}{c}-23.9 \% \\
(0.04) \\
\end{array}$ & $\begin{array}{l}69.9 \% \\
(0.06) \\
\end{array}$ & $\begin{array}{c}-11.9 \% \\
(0.06) \\
\end{array}$ & $\begin{array}{l}82.7 \% \\
(0.07) \\
\end{array}$ & $\begin{array}{l}-1.9 \% \\
(0.05) \\
\end{array}$ & $\begin{array}{l}87.4 \% \\
(0.08) \\
\end{array}$ & $\begin{array}{r}1.5 \% \\
(0.06) \\
\end{array}$ & $\begin{array}{l}87.5 \% \\
(0.08) \\
\end{array}$ & $\begin{array}{l}0.1 \% \\
(0.06) \\
\end{array}$ & $\begin{array}{l}84.7 \% \\
(0.09) \\
\end{array}$ & $\begin{array}{l}-4.8 \% \\
(0.06) \\
\end{array}$ & $\begin{array}{c}93.1 \% \\
(0.1) \\
\end{array}$ & $\begin{array}{l}-3.0 \% \\
(0.06) \\
\end{array}$ & \begin{tabular}{|c|}
$108.9 \%$ \\
$(0.16)$
\end{tabular} & $\begin{array}{l}28.4 \% \\
(0.08) \\
\end{array}$ & $\begin{array}{c}94.5 \% \\
(0.2) \\
\end{array}$ & $\begin{array}{l}33.5 \% \\
(0.09) \\
\end{array}$ \\
\hline Brazil & $112.5 \%$ & $27.0 \%$ & $\begin{array}{l}80.8 \% \\
(0.02) \\
\end{array}$ & $\begin{array}{l}17.7 \% \\
(0.01) \\
\end{array}$ & $\begin{array}{l}70.3 \% \\
(0.02) \\
\end{array}$ & $\begin{array}{l}21.6 \% \\
(0.01) \\
\end{array}$ & $\begin{array}{l}80.4 \% \\
(0.02) \\
\end{array}$ & $\begin{array}{l}26.5 \% \\
(0.02) \\
\end{array}$ & $\begin{array}{l}78.6 \% \\
(0.02) \\
\end{array}$ & $\begin{array}{l}25.9 \% \\
(0.02) \\
\end{array}$ & $\begin{array}{l}78.2 \% \\
(0.02) \\
\end{array}$ & $\begin{array}{l}25.8 \% \\
(0.02) \\
\end{array}$ & $\begin{array}{l}79.2 \% \\
(0.02) \\
\end{array}$ & $\begin{array}{l}24.9 \% \\
(0.02) \\
\end{array}$ & $\begin{array}{l}77.7 \% \\
(0.02) \\
\end{array}$ & $\begin{array}{l}25.4 \% \\
(0.02) \\
\end{array}$ & \begin{tabular}{|c|}
$100.9 \%$ \\
$(0.03)$ \\
\end{tabular} & $\begin{array}{l}37.0 \% \\
(0.02) \\
\end{array}$ & $\begin{array}{l}97.2 \% \\
(0.03) \\
\end{array}$ & $\begin{array}{l}37.8 \% \\
(0.02) \\
\end{array}$ \\
\hline Chile & $45.4 \%$ & $36.3 \%$ & $\begin{array}{l}33.1 \% \\
(0.02) \\
\end{array}$ & $\begin{array}{l}24.8 \% \\
(0.02) \\
\end{array}$ & $\begin{array}{l}39.4 \% \\
(0.03) \\
\end{array}$ & $\begin{array}{l}22.5 \% \\
(0.03) \\
\end{array}$ & $\begin{array}{l}50.2 \% \\
(0.04) \\
\end{array}$ & $\begin{array}{l}26.7 \% \\
(0.05) \\
\end{array}$ & $\begin{array}{l}50.6 \% \\
(0.05) \\
\end{array}$ & $\begin{array}{l}26.2 \% \\
(0.05) \\
\end{array}$ & $\begin{array}{l}48.7 \% \\
(0.05) \\
\end{array}$ & $\begin{array}{l}24.0 \% \\
(0.05) \\
\end{array}$ & $\begin{array}{l}43.3 \% \\
(0.05) \\
\end{array}$ & $\begin{array}{l}21.4 \% \\
(0.05) \\
\end{array}$ & $\begin{array}{l}42.9 \% \\
(0.05) \\
\end{array}$ & $\begin{array}{l}19.6 \% \\
(0.05) \\
\end{array}$ & $\begin{array}{l}54.9 \% \\
(0.06) \\
\end{array}$ & $\begin{array}{l}19.4 \% \\
(0.05) \\
\end{array}$ & $\begin{array}{l}52.0 \% \\
(0.06) \\
\end{array}$ & $\begin{array}{l}17.9 \% \\
(0.05) \\
\end{array}$ \\
\hline Costa Rica & $-4.6 \%$ & $-3.6 \%$ & $\begin{array}{l}-8.4 \% \\
(0.03) \\
\end{array}$ & $\begin{array}{l}-1.0 \% \\
(0.03) \\
\end{array}$ & $\begin{array}{l}-10.2 \% \\
(0.05) \\
\end{array}$ & $\begin{array}{l}-1.0 \% \\
(0.05) \\
\end{array}$ & $\begin{array}{l}-2.7 \% \\
(0.06) \\
\end{array}$ & $\begin{array}{r}9.5 \% \\
(0.07) \\
\end{array}$ & $\begin{array}{c}-11.4 \% \\
(0.06) \\
\end{array}$ & $\begin{array}{r}9.3 \% \\
(0.06) \\
\end{array}$ & $\begin{array}{l}-8.7 \% \\
(0.07) \\
\end{array}$ & $\begin{array}{l}10.5 \% \\
(0.06) \\
\end{array}$ & $\begin{array}{c}-11.9 \% \\
(0.06) \\
\end{array}$ & $\begin{array}{l}11.0 \% \\
(0.06) \\
\end{array}$ & $\begin{array}{r}-14.8 \% \\
(0.07) \\
\end{array}$ & $\begin{array}{l}8.5 \% \\
(0.06) \\
\end{array}$ & $\begin{array}{c}-17.9 \% \\
(0.06) \\
\end{array}$ & $\begin{array}{l}13.8 \% \\
(0.07) \\
\end{array}$ & $\begin{array}{l}-15.4 \% \\
(0.06) \\
\end{array}$ & $\begin{array}{l}18.9 \% \\
(0.08) \\
\end{array}$ \\
\hline Dom. Rep. & 78.1\% & $25.5 \%$ & $\begin{array}{l}62.2 \% \\
(0.06) \\
\end{array}$ & $\begin{array}{l}19.3 \% \\
(0.04) \\
\end{array}$ & $\begin{array}{l}63.8 \% \\
(0.12) \\
\end{array}$ & $\begin{array}{l}24.1 \% \\
(0.08) \\
\end{array}$ & $\begin{array}{l}55.4 \% \\
(0.08) \\
\end{array}$ & $\begin{array}{l}32.7 \% \\
(0.09) \\
\end{array}$ & $\begin{array}{l}55.3 \% \\
(0.08) \\
\end{array}$ & $\begin{array}{l}36.6 \% \\
(0.11) \\
\end{array}$ & $\begin{array}{l}52.7 \% \\
(0.09) \\
\end{array}$ & $\begin{array}{c}25.0 \% \\
(0.1) \\
\end{array}$ & $\begin{array}{l}47.2 \% \\
(0.09) \\
\end{array}$ & $\begin{array}{l}15.0 \% \\
(0.12) \\
\end{array}$ & $\begin{array}{l}52.8 \% \\
(0.11) \\
\end{array}$ & $\begin{array}{l}18.2 \% \\
(0.12) \\
\end{array}$ & $\begin{array}{l}59.8 \% \\
(0.16) \\
\end{array}$ & $\begin{array}{c}25.6 \% \\
(0.2) \\
\end{array}$ & $\begin{array}{l}43.2 \% \\
(0.16) \\
\end{array}$ & $\begin{array}{l}23.2 \% \\
(0.26) \\
\end{array}$ \\
\hline Ecuador & $9.0 \%$ & $26.3 \%$ & $\begin{array}{c}8.8 \% \\
(0.05) \\
\end{array}$ & $\begin{array}{l}23.7 \% \\
(0.03) \\
\end{array}$ & $\begin{array}{c}7.9 \% \\
(0.06) \\
\end{array}$ & $\begin{array}{l}26.6 \% \\
(0.04) \\
\end{array}$ & $\begin{array}{c}9.4 \% \\
(0.06) \\
\end{array}$ & $\begin{array}{l}14.1 \% \\
(0.04) \\
\end{array}$ & $\begin{array}{c}8.6 \% \\
(0.06) \\
\end{array}$ & $\begin{array}{l}17.3 \% \\
(0.05) \\
\end{array}$ & $\begin{array}{c}8.9 \% \\
(0.07) \\
\end{array}$ & $\begin{array}{l}19.9 \% \\
(0.05) \\
\end{array}$ & $\begin{array}{l}12.0 \% \\
(0.08) \\
\end{array}$ & $\begin{array}{l}16.9 \% \\
(0.05) \\
\end{array}$ & $\begin{array}{l}10.7 \% \\
(0.11) \\
\end{array}$ & $\begin{array}{l}17.4 \% \\
(0.05) \\
\end{array}$ & $\begin{array}{l}29.1 \% \\
(0.18) \\
\end{array}$ & $\begin{array}{l}35.3 \% \\
(0.07) \\
\end{array}$ & $\begin{array}{l}33.5 \% \\
(0.19) \\
\end{array}$ & $\begin{array}{l}28.4 \% \\
(0.08) \\
\end{array}$ \\
\hline El Salvador & $-1.9 \%$ & $-5.3 \%$ & $\begin{array}{l}-4.6 \% \\
(0.03) \\
\end{array}$ & $\begin{array}{l}-5.9 \% \\
(0.02) \\
\end{array}$ & $\begin{array}{l}-2.7 \% \\
(0.04) \\
\end{array}$ & $\begin{array}{l}-0.3 \% \\
(0.05) \\
\end{array}$ & $\begin{array}{l}-6.4 \% \\
(0.04) \\
\end{array}$ & $\begin{array}{c}-10.1 \% \\
(0.06) \\
\end{array}$ & $\begin{array}{l}-7.0 \% \\
(0.04) \\
\end{array}$ & $\begin{array}{l}-6.9 \% \\
(0.06) \\
\end{array}$ & $\begin{array}{l}-8.2 \% \\
(0.04) \\
\end{array}$ & $\begin{array}{c}-13.0 \% \\
(0.06) \\
\end{array}$ & $\begin{array}{l}-6.2 \% \\
(0.05) \\
\end{array}$ & $\begin{array}{c}-11.7 \% \\
(0.06) \\
\end{array}$ & $\begin{array}{l}-7.1 \% \\
(0.05) \\
\end{array}$ & $\begin{array}{c}-17.2 \% \\
(0.05) \\
\end{array}$ & $\begin{array}{l}12.1 \% \\
(0.09) \\
\end{array}$ & $\begin{array}{l}4.6 \% \\
(0.1) \\
\end{array}$ & $\begin{array}{l}11.3 \% \\
(0.11) \\
\end{array}$ & $\begin{array}{l}5.6 \% \\
(0.1) \\
\end{array}$ \\
\hline Honduras & $-0.9 \%$ & $-17.9 \%$ & $\begin{array}{r}-10.1 \% \\
(0.04) \\
\end{array}$ & $\begin{array}{c}-21.0 \% \\
(0.03) \\
\end{array}$ & $\begin{array}{c}-11.7 \% \\
(0.04) \\
\end{array}$ & $\begin{array}{c}-23.1 \% \\
(0.03) \\
\end{array}$ & $\begin{array}{r}-10.6 \% \\
(0.04) \\
\end{array}$ & $\begin{array}{r}-20.2 \% \\
(0.04) \\
\end{array}$ & $\begin{array}{l}-9.6 \% \\
(0.04) \\
\end{array}$ & $\begin{array}{c}-18.7 \% \\
(0.04) \\
\end{array}$ & $\begin{array}{r}-9.7 \% \\
(0.04) \\
\end{array}$ & $\begin{array}{c}-18.4 \% \\
(0.04) \\
\end{array}$ & $\begin{array}{l}-6.5 \% \\
(0.04) \\
\end{array}$ & $\begin{array}{c}-12.7 \% \\
(0.05) \\
\end{array}$ & $\begin{array}{l}-5.5 \% \\
(0.05) \\
\end{array}$ & $\begin{array}{l}-9.1 \% \\
(0.05) \\
\end{array}$ & $\begin{array}{c}9.5 \% \\
(0.07) \\
\end{array}$ & $\begin{array}{c}33.4 \% \\
(0.1) \\
\end{array}$ & $\begin{array}{r}8.5 \% \\
(0.07) \\
\end{array}$ & $\begin{array}{l}36.3 \% \\
(0.12) \\
\end{array}$ \\
\hline Nicaragua & $112.4 \%$ & $98.8 \%$ & $\begin{array}{l}90.8 \% \\
(0.08) \\
\end{array}$ & $\begin{array}{l}91.2 \% \\
(0.06) \\
\end{array}$ & $\begin{array}{c}80.1 \% \\
(0.1) \\
\end{array}$ & $\begin{array}{l}84.3 \% \\
(0.07) \\
\end{array}$ & $\begin{array}{l}65.3 \% \\
(0.11) \\
\end{array}$ & $\begin{array}{l}56.2 \% \\
(0.08) \\
\end{array}$ & $\begin{array}{c}52.5 \% \\
(0.1) \\
\end{array}$ & $\begin{array}{l}51.8 \% \\
(0.09) \\
\end{array}$ & $\begin{array}{l}70.6 \% \\
(0.17) \\
\end{array}$ & $\begin{array}{l}50.1 \% \\
(0.11) \\
\end{array}$ & $\begin{array}{l}71.3 \% \\
(0.19) \\
\end{array}$ & $\begin{array}{l}43.5 \% \\
(0.11) \\
\end{array}$ & $\begin{array}{l}66.9 \% \\
(0.21) \\
\end{array}$ & $\begin{array}{l}32.9 \% \\
(0.11) \\
\end{array}$ & $\begin{array}{c}139.6 \% \\
(0.42) \\
\end{array}$ & $\begin{array}{l}49.3 \% \\
(0.16) \\
\end{array}$ & $\begin{array}{c}151.8 \% \\
(0.45) \\
\end{array}$ & $\begin{array}{l}57.3 \% \\
(0.16) \\
\end{array}$ \\
\hline Panama & $37.5 \%$ & $20.1 \%$ & $\begin{array}{l}30.4 \% \\
(0.03) \\
\end{array}$ & $\begin{array}{l}17.2 \% \\
(0.03) \\
\end{array}$ & $\begin{array}{l}34.2 \% \\
(0.05) \\
\end{array}$ & $\begin{array}{l}19.1 \% \\
(0.04) \\
\end{array}$ & $\begin{array}{l}22.9 \% \\
(0.06) \\
\end{array}$ & $\begin{array}{l}22.0 \% \\
(0.05) \\
\end{array}$ & $\begin{array}{l}21.2 \% \\
(0.06) \\
\end{array}$ & $\begin{array}{l}18.3 \% \\
(0.05) \\
\end{array}$ & $\begin{array}{l}17.7 \% \\
(0.06) \\
\end{array}$ & $\begin{array}{l}20.6 \% \\
(0.06) \\
\end{array}$ & $\begin{array}{l}13.5 \% \\
(0.07) \\
\end{array}$ & $\begin{array}{l}23.7 \% \\
(0.06) \\
\end{array}$ & $\begin{array}{l}16.7 \% \\
(0.08) \\
\end{array}$ & $\begin{array}{l}22.5 \% \\
(0.06) \\
\end{array}$ & $\begin{array}{l}29.2 \% \\
(0.11) \\
\end{array}$ & $\begin{array}{l}25.8 \% \\
(0.07) \\
\end{array}$ & $\begin{array}{c}24.1 \% \\
(0.1) \\
\end{array}$ & $\begin{array}{l}24.6 \% \\
(0.07) \\
\end{array}$ \\
\hline Paraguay & $75.0 \%$ & $39.3 \%$ & $\begin{array}{l}62.9 \% \\
(0.08) \\
\end{array}$ & $\begin{array}{l}46.4 \% \\
(0.05) \\
\end{array}$ & $\begin{array}{l}33.3 \% \\
(0.12) \\
\end{array}$ & $\begin{array}{l}45.6 \% \\
(0.09) \\
\end{array}$ & $\begin{array}{c}4.0 \% \\
(0.12) \\
\end{array}$ & $\begin{array}{l}17.1 \% \\
(0.09) \\
\end{array}$ & $\begin{array}{r}6.1 \% \\
(0.13) \\
\end{array}$ & $\begin{array}{c}6.0 \% \\
(0.08) \\
\end{array}$ & $\begin{array}{r}5.8 \% \\
(0.13) \\
\end{array}$ & $\begin{array}{l}-6.9 \% \\
(0.08) \\
\end{array}$ & $\begin{array}{l}10.8 \% \\
(0.15) \\
\end{array}$ & $\begin{array}{l}-0.5 \% \\
(0.09) \\
\end{array}$ & $\begin{array}{c}-4.4 \% \\
(0.1) \\
\end{array}$ & $\begin{array}{c}4.0 \% \\
(0.11) \\
\end{array}$ & $\begin{array}{r}3.5 \% \\
(0.18) \\
\end{array}$ & $\begin{array}{c}3.3 \% \\
(0.15) \\
\end{array}$ & $\begin{array}{r}2.4 \% \\
(0.24) \\
\end{array}$ & $\begin{array}{l}-3.4 \% \\
(0.09) \\
\end{array}$ \\
\hline Peru & $36.4 \%$ & $33.7 \%$ & $\begin{array}{l}29.0 \% \\
(0.04) \\
\end{array}$ & $\begin{array}{l}24.5 \% \\
(0.03) \\
\end{array}$ & $\begin{array}{c}35.6 \% \\
(0.1) \\
\end{array}$ & $\begin{array}{l}33.0 \% \\
(0.04) \\
\end{array}$ & $\begin{array}{l}33.5 \% \\
(0.09) \\
\end{array}$ & $\begin{array}{l}37.8 \% \\
(0.04) \\
\end{array}$ & $\begin{array}{l}29.5 \% \\
(0.12) \\
\end{array}$ & $\begin{array}{l}35.0 \% \\
(0.05) \\
\end{array}$ & $\begin{array}{l}29.9 \% \\
(0.12) \\
\end{array}$ & $\begin{array}{l}36.8 \% \\
(0.05) \\
\end{array}$ & $\begin{array}{l}29.7 \% \\
(0.12) \\
\end{array}$ & $\begin{array}{l}29.2 \% \\
(0.05) \\
\end{array}$ & $\begin{array}{l}25.4 \% \\
(0.12) \\
\end{array}$ & $\begin{array}{l}26.1 \% \\
(0.05) \\
\end{array}$ & $\begin{array}{l}43.8 \% \\
(0.21) \\
\end{array}$ & $\begin{array}{l}45.6 \% \\
(0.07) \\
\end{array}$ & $\begin{array}{l}50.3 \% \\
(0.26) \\
\end{array}$ & $\begin{array}{l}42.4 \% \\
(0.08) \\
\end{array}$ \\
\hline Uruguay & $42.9 \%$ & $25.6 \%$ & $\begin{array}{l}30.6 \% \\
(0.03) \\
\end{array}$ & $\begin{array}{l}30.7 \% \\
(0.04) \\
\end{array}$ & $\begin{array}{l}33.1 \% \\
(0.04) \\
\end{array}$ & $\begin{array}{l}26.6 \% \\
(0.04) \\
\end{array}$ & $\begin{array}{l}57.5 \% \\
(0.05) \\
\end{array}$ & $\begin{array}{l}-7.9 \% \\
(0.04) \\
\end{array}$ & $\begin{array}{r}59.8 \% \\
(0.05) \\
\end{array}$ & $\begin{array}{l}-9.1 \% \\
(0.04) \\
\end{array}$ & $\begin{array}{l}62.5 \% \\
(0.06) \\
\end{array}$ & $\begin{array}{r}-8.6 \% \\
(0.04) \\
\end{array}$ & $\begin{array}{l}70.8 \% \\
(0.07) \\
\end{array}$ & $\begin{array}{c}-12.8 \% \\
(0.05) \\
\end{array}$ & $\begin{array}{l}66.3 \% \\
(0.08) \\
\end{array}$ & $\begin{array}{l}-4.0 \% \\
(0.06) \\
\end{array}$ & $\begin{array}{l}81.8 \% \\
(0.09) \\
\end{array}$ & $\begin{array}{l}17.0 \% \\
(0.08) \\
\end{array}$ & $\begin{array}{c}67.8 \% \\
(0.1) \\
\end{array}$ & $\begin{array}{l}19.0 \% \\
(0.11) \\
\end{array}$ \\
\hline $\begin{array}{l}\text { Latin America } \\
\text { (13 countries) }\end{array}$ & $80.5 \%$ & $23.3 \%$ & $\begin{array}{l}67.2 \% \\
(0.01) \\
\end{array}$ & $\begin{array}{l}17.9 \% \\
(0.01) \\
\end{array}$ & $\begin{array}{l}60.4 \% \\
(0.01) \\
\end{array}$ & $\begin{array}{l}21.5 \% \\
(0.01) \\
\end{array}$ & $\begin{array}{l}68.5 \% \\
(0.01) \\
\end{array}$ & $\begin{array}{l}24.0 \% \\
(0.01) \\
\end{array}$ & $\begin{array}{l}67.6 \% \\
(0.01) \\
\end{array}$ & $\begin{array}{l}23.7 \% \\
(0.01) \\
\end{array}$ & $\begin{array}{l}68.0 \% \\
(0.01) \\
\end{array}$ & $\begin{array}{l}23.5 \% \\
(0.01) \\
\end{array}$ & $\begin{array}{l}68.9 \% \\
(0.02) \\
\end{array}$ & $\begin{array}{l}22.0 \% \\
(0.01) \\
\end{array}$ & $\begin{array}{l}68.4 \% \\
(0.02) \\
\end{array}$ & $\begin{array}{l}22.5 \% \\
(0.01) \\
\end{array}$ & $\begin{array}{l}92.7 \% \\
(0.02) \\
\end{array}$ & $\begin{array}{l}35.1 \% \\
(0.02) \\
\end{array}$ & $\begin{array}{l}89.4 \% \\
(0.02) \\
\end{array}$ & $\begin{array}{l}35.3 \% \\
(0.02) \\
\end{array}$ \\
\hline
\end{tabular}




\begin{tabular}{|c|c|c|c|c|c|c|c|c|c|c|c|c|c|c|c|c|c|c|c|c|}
\hline \multicolumn{21}{|c|}{ High School Teachers vis-à-vis Other Professionals and Technicians } \\
\hline \multirow[t]{2}{*}{ Country } & \multicolumn{2}{|c|}{ Original gap } & \multicolumn{2}{|c|}{ +Gender } & \multicolumn{2}{|c|}{+ Age } & \multicolumn{2}{|c|}{ + Education } & \multicolumn{2}{|c|}{$\begin{array}{c}\text { + Children } \\
\text { living in the } \\
\text { household }\end{array}$} & \multicolumn{2}{|c|}{\begin{tabular}{|c|}
$\begin{array}{c}\text { Elders living } \\
\text { in the } \\
\text { household }\end{array}$ \\
\end{tabular}} & \multicolumn{2}{|c|}{$\begin{array}{c}\text { + Household } \\
\text { head }\end{array}$} & \multicolumn{2}{|c|}{$\begin{array}{l}\text { + Another wage } \\
\text { earner living in } \\
\text { the household }\end{array}$} & \multicolumn{2}{|c|}{$\begin{array}{c}\text { + Part-time } \\
\text { Work }\end{array}$} & \multicolumn{2}{|c|}{$\begin{array}{c}\text { + Has more than } \\
\text { one job }\end{array}$} \\
\hline & C-97 & C-07 & C-97 & C-07 & C-97 & C-07 & C-97 & C-07 & C-97 & C-07 & C-97 & C-07 & C-97 & C-07 & C-97 & C-07 & C-97 & C-07 & C-97 & C-07 \\
\hline Bolivia & $35.1 \%$ & $-17.9 \%$ & $\begin{array}{l}30.7 \% \\
(0.05)\end{array}$ & $\begin{array}{c}-18.9 \% \\
(0.04)\end{array}$ & $\begin{array}{l}35.1 \% \\
(0.08)\end{array}$ & $\begin{array}{c}-12.0 \% \\
(0.07)\end{array}$ & $\begin{array}{l}43.2 \% \\
(0.08)\end{array}$ & $\begin{array}{l}-2.6 \% \\
(0.07)\end{array}$ & $\begin{array}{l}43.0 \% \\
(0.09)\end{array}$ & $\begin{array}{l}-1.1 \% \\
(0.07)\end{array}$ & \begin{tabular}{|l|}
$48.6 \%$ \\
$(0.09)$ \\
\end{tabular} & $\begin{array}{l}-0.7 \% \\
(0.07)\end{array}$ & $\begin{array}{l}43.3 \% \\
(0.11)\end{array}$ & $\begin{array}{l}5.5 \% \\
(0.08)\end{array}$ & $\begin{array}{l}43.3 \% \\
(0.12)\end{array}$ & $\begin{array}{c}0.0 \% \\
(0.07)\end{array}$ & $\begin{array}{l}60.1 \% \\
(0.21)\end{array}$ & $\begin{array}{l}13.7 \% \\
(0.11)\end{array}$ & $\begin{array}{l}61.4 \% \\
(0.24)\end{array}$ & $\begin{array}{l}17.1 \% \\
(0.11)\end{array}$ \\
\hline Brazil & $23.9 \%$ & $-3.2 \%$ & $\begin{array}{l}15.5 \% \\
(0.04) \\
\end{array}$ & $\begin{array}{l}-6.5 \% \\
(0.02) \\
\end{array}$ & $\begin{array}{l}12.9 \% \\
(0.05) \\
\end{array}$ & $\begin{array}{l}0.6 \% \\
(0.03) \\
\end{array}$ & $\begin{array}{l}25.8 \% \\
(0.06) \\
\end{array}$ & $\begin{array}{c}9.7 \% \\
(0.04)\end{array}$ & $\begin{array}{l}25.0 \% \\
(0.06) \\
\end{array}$ & $\begin{array}{l}10.6 \% \\
(0.05) \\
\end{array}$ & \begin{tabular}{|l|}
$23.2 \%$ \\
$(0.06)$ \\
\end{tabular} & $\begin{array}{l}10.6 \% \\
(0.05) \\
\end{array}$ & $\begin{array}{l}22.9 \% \\
(0.06) \\
\end{array}$ & $\begin{array}{c}9.5 \% \\
(0.05) \\
\end{array}$ & $\begin{array}{l}25.4 \% \\
(0.06)\end{array}$ & $\begin{array}{l}8.9 \% \\
(0.05) \\
\end{array}$ & $\begin{array}{l}29.1 \% \\
(0.06)\end{array}$ & $\begin{array}{l}14.2 \% \\
(0.05) \\
\end{array}$ & $\begin{array}{l}27.1 \% \\
(0.07) \\
\end{array}$ & $\begin{array}{l}16.4 \% \\
(0.06) \\
\end{array}$ \\
\hline Chile & $26.2 \%$ & $5.6 \%$ & $\begin{array}{l}21.1 \% \\
(0.04) \\
\end{array}$ & $\begin{array}{l}1.8 \% \\
(0.04) \\
\end{array}$ & $\begin{array}{l}28.5 \% \\
(0.06) \\
\end{array}$ & $\begin{array}{l}4.6 \% \\
(0.08) \\
\end{array}$ & $\begin{array}{l}61.2 \% \\
(0.09) \\
\end{array}$ & $\begin{array}{l}21.7 \% \\
(0.13) \\
\end{array}$ & $\begin{array}{l}57.7 \% \\
(0.09) \\
\end{array}$ & $\begin{array}{l}19.1 \% \\
(0.11) \\
\end{array}$ & \begin{tabular}{|l|}
$55.0 \%$ \\
$(0.09)$ \\
\end{tabular} & $\begin{array}{c}16.2 \% \\
(0.1) \\
\end{array}$ & $\begin{array}{l}51.3 \% \\
(0.09) \\
\end{array}$ & $\begin{array}{c}13.8 \% \\
(0.1) \\
\end{array}$ & \begin{tabular}{|l|}
$50.7 \%$ \\
$(0.09)$ \\
\end{tabular} & $\begin{array}{l}16.5 \% \\
(0.09) \\
\end{array}$ & $\begin{array}{l}54.8 \% \\
(0.11) \\
\end{array}$ & $\begin{array}{l}17.5 \% \\
(0.09) \\
\end{array}$ & \begin{tabular}{|l|}
$52.2 \%$ \\
$(0.11)$ \\
\end{tabular} & $\begin{array}{l}17.6 \% \\
(0.08) \\
\end{array}$ \\
\hline Costa Rica & $-12.0 \%$ & $-13.6 \%$ & \begin{tabular}{|c|}
$-14.3 \%$ \\
$(0.05)$ \\
\end{tabular} & $\begin{array}{c}-12.3 \% \\
(0.04) \\
\end{array}$ & \begin{tabular}{|c|}
$-16.5 \%$ \\
$(0.09)$ \\
\end{tabular} & $\begin{array}{l}-8.9 \% \\
(0.09) \\
\end{array}$ & $\begin{array}{l}-2.4 \% \\
(0.13) \\
\end{array}$ & $\begin{array}{c}2.3 \% \\
(0.12) \\
\end{array}$ & $\begin{array}{l}-7.0 \% \\
(0.15) \\
\end{array}$ & $\begin{array}{c}3.5 \% \\
(0.11) \\
\end{array}$ & \begin{tabular}{|c|}
$-13.8 \%$ \\
$(0.15)$ \\
\end{tabular} & $\begin{array}{l}0.8 \% \\
(0.1) \\
\end{array}$ & \begin{tabular}{|c|}
$-13.2 \%$ \\
$(0.15)$ \\
\end{tabular} & $\begin{array}{l}2.9 \% \\
(0.1) \\
\end{array}$ & \begin{tabular}{|c|}
$-15.2 \%$ \\
$(0.17)$ \\
\end{tabular} & $\begin{array}{c}5.1 \% \\
(0.11)\end{array}$ & $\begin{array}{l}1.3 \% \\
(0.23) \\
\end{array}$ & $\begin{array}{l}17.9 \% \\
(0.13) \\
\end{array}$ & \begin{tabular}{|c|}
$-10.7 \%$ \\
$(0.26)$ \\
\end{tabular} & $\begin{array}{l}13.6 \% \\
(0.11) \\
\end{array}$ \\
\hline Dom. Rep. & $24.9 \%$ & $13.1 \%$ & $\begin{array}{l}22.9 \% \\
(0.18) \\
\end{array}$ & $\begin{array}{l}11.7 \% \\
(0.07) \\
\end{array}$ & $\begin{array}{l}26.1 \% \\
(0.22) \\
\end{array}$ & $\begin{array}{l}12.6 \% \\
(0.23) \\
\end{array}$ & $\begin{array}{l}26.4 \% \\
(0.21) \\
\end{array}$ & $\begin{array}{l}29.2 \% \\
(0.24) \\
\end{array}$ & $\begin{array}{l}30.9 \% \\
(0.28) \\
\end{array}$ & $\begin{array}{l}25.2 \% \\
(0.23) \\
\end{array}$ & $\begin{array}{l}34.2 \% \\
(0.31) \\
\end{array}$ & $\begin{array}{l}13.2 \% \\
(0.25) \\
\end{array}$ & $\begin{array}{l}28.2 \% \\
(0.31) \\
\end{array}$ & $\begin{array}{c}7.6 \% \\
(0.28) \\
\end{array}$ & $\begin{array}{l}19.2 \% \\
(0.28) \\
\end{array}$ & $\begin{array}{c}20.8 \% \\
(0.3) \\
\end{array}$ & $\begin{array}{l}18.0 \% \\
(0.42) \\
\end{array}$ & $\begin{array}{l}16.0 \% \\
(0.31) \\
\end{array}$ & $\begin{array}{l}66.0 \% \\
(0.58) \\
\end{array}$ & $\begin{array}{c}4.8 \% \\
(0.38) \\
\end{array}$ \\
\hline Ecuador & $-7.0 \%$ & $-4.5 \%$ & $\begin{array}{l}-7.1 \% \\
(0.11) \\
\end{array}$ & $\begin{array}{l}-5.7 \% \\
(0.04) \\
\end{array}$ & $\begin{array}{c}-15.4 \% \\
(0.18) \\
\end{array}$ & $\begin{array}{c}9.6 \% \\
(0.07) \\
\end{array}$ & \begin{tabular}{|c|}
$-13.2 \%$ \\
$(0.2)$ \\
\end{tabular} & $\begin{array}{l}14.0 \% \\
(0.08) \\
\end{array}$ & $\begin{array}{c}-16.1 \% \\
(0.21) \\
\end{array}$ & $\begin{array}{l}12.2 \% \\
(0.07) \\
\end{array}$ & $\begin{array}{c}-14.4 \% \\
(0.31) \\
\end{array}$ & $\begin{array}{l}16.5 \% \\
(0.08) \\
\end{array}$ & \begin{tabular}{|c|}
$-38.4 \%$ \\
$(0.27)$ \\
\end{tabular} & $\begin{array}{l}15.9 \% \\
(0.08) \\
\end{array}$ & $\begin{array}{l}-0.3 \% \\
(0.33) \\
\end{array}$ & $\begin{array}{l}16.4 \% \\
(0.09) \\
\end{array}$ & $\begin{array}{l}20.4 \% \\
(0.74) \\
\end{array}$ & $\begin{array}{l}41.4 \% \\
(0.12) \\
\end{array}$ & $\begin{array}{l}28.3 \% \\
(0.17) \\
\end{array}$ & $\begin{array}{l}29.3 \% \\
(0.13) \\
\end{array}$ \\
\hline El Salvador & $-0.9 \%$ & $7.8 \%$ & $\begin{array}{l}-0.7 \% \\
(0.12) \\
\end{array}$ & $\begin{array}{c}7.6 \% \\
(0.06) \\
\end{array}$ & $\begin{array}{c}-2.4 \% \\
(0.2) \\
\end{array}$ & $\begin{array}{c}6.5 \% \\
(0.23) \\
\end{array}$ & $\begin{array}{c}7.7 \% \\
(0.21) \\
\end{array}$ & $\begin{array}{l}33.7 \% \\
(0.58) \\
\end{array}$ & $\begin{array}{c}4.2 \% \\
(0.24) \\
\end{array}$ & $\begin{array}{c}7.0 \% \\
(0.22) \\
\end{array}$ & \begin{tabular}{|c|}
$3.6 \%$ \\
$(0.22)$ \\
\end{tabular} & $\begin{array}{l}-4.9 \% \\
(0.19) \\
\end{array}$ & $\begin{array}{c}2.9 \% \\
(0.21) \\
\end{array}$ & $\begin{array}{l}-8.0 \% \\
(0.19) \\
\end{array}$ & \begin{tabular}{|c|}
$8.6 \%$ \\
$(0.26)$ \\
\end{tabular} & $\begin{array}{c}-4.5 \% \\
(0.2) \\
\end{array}$ & $\begin{array}{l}-6.8 \% \\
(0.15) \\
\end{array}$ & $\begin{array}{c}-13.5 \% \\
(0.23)\end{array}$ & $\begin{array}{l}-1.6 \% \\
(0.18) \\
\end{array}$ & $\begin{array}{l}-5.8 \% \\
(0.16) \\
\end{array}$ \\
\hline Honduras & $-22.2 \%$ & $-19.0 \%$ & $\begin{array}{c}-23.2 \% \\
(0.06) \\
\end{array}$ & $\begin{array}{c}-20.1 \% \\
(0.04) \\
\end{array}$ & $\begin{array}{c}-18.0 \% \\
(0.08) \\
\end{array}$ & $\begin{array}{c}-14.7 \% \\
(0.07) \\
\end{array}$ & $\begin{array}{c}0.8 \% \\
(0.11) \\
\end{array}$ & $\begin{array}{l}8.4 \% \\
(0.1) \\
\end{array}$ & $\begin{array}{c}-11.8 \% \\
(0.09) \\
\end{array}$ & $\begin{array}{c}4.9 \% \\
(0.09) \\
\end{array}$ & $\begin{array}{c}-17.9 \% \\
(0.1) \\
\end{array}$ & $\begin{array}{c}3.6 \% \\
(0.09) \\
\end{array}$ & $\begin{array}{c}-16.0 \% \\
(0.1) \\
\end{array}$ & $\begin{array}{c}10.2 \% \\
(0.1) \\
\end{array}$ & $\begin{array}{c}-12.5 \% \\
(0.11) \\
\end{array}$ & $\begin{array}{l}9.6 \% \\
(0.1) \\
\end{array}$ & $\begin{array}{l}-7.5 \% \\
(0.09) \\
\end{array}$ & $\begin{array}{l}30.9 \% \\
(0.17) \\
\end{array}$ & $\begin{array}{l}-2.4 \% \\
(0.09) \\
\end{array}$ & $\begin{array}{l}12.0 \% \\
(0.13) \\
\end{array}$ \\
\hline Nicaragua & $28.9 \%$ & $60.9 \%$ & $\begin{array}{l}23.4 \% \\
(0.16) \\
\end{array}$ & $\begin{array}{l}58.9 \% \\
(0.08) \\
\end{array}$ & $\begin{array}{l}51.0 \% \\
(0.29) \\
\end{array}$ & $\begin{array}{l}45.6 \% \\
(0.17) \\
\end{array}$ & $\begin{array}{c}111.5 \% \\
(0.33) \\
\end{array}$ & $\begin{array}{l}70.9 \% \\
(0.25) \\
\end{array}$ & $\begin{array}{c}125.6 \% \\
(0.4)\end{array}$ & $\begin{array}{l}63.5 \% \\
(0.23) \\
\end{array}$ & $\begin{array}{c}21.0 \% \\
(0.42) \\
\end{array}$ & $\begin{array}{l}74.3 \% \\
(0.28) \\
\end{array}$ & $\begin{array}{c}132.2 \% \\
(0.58) \\
\end{array}$ & $\begin{array}{l}59.9 \% \\
(0.25) \\
\end{array}$ & $\begin{array}{l}47.3 \% \\
(0.83) \\
\end{array}$ & $\begin{array}{l}42.5 \% \\
(0.33) \\
\end{array}$ & $\begin{array}{c}28.2 \% \\
(0) \\
\end{array}$ & $\begin{array}{l}58.6 \% \\
(0.38) \\
\end{array}$ & $\begin{array}{c}28.2 \% \\
(0) \\
\end{array}$ & $\begin{array}{l}51.5 \% \\
(0.42) \\
\end{array}$ \\
\hline Panama & $-0.6 \%$ & $-3.7 \%$ & $\begin{array}{l}-2.1 \% \\
(0.03) \\
\end{array}$ & $\begin{array}{l}-4.3 \% \\
(0.03) \\
\end{array}$ & $\begin{array}{c}9.2 \% \\
(0.06) \\
\end{array}$ & $\begin{array}{l}-2.8 \% \\
(0.05) \\
\end{array}$ & $\begin{array}{l}24.8 \% \\
(0.07) \\
\end{array}$ & $\begin{array}{l}15.8 \% \\
(0.06) \\
\end{array}$ & $\begin{array}{l}23.0 \% \\
(0.08) \\
\end{array}$ & $\begin{array}{l}16.1 \% \\
(0.06) \\
\end{array}$ & $\begin{array}{l}22.8 \% \\
(0.07) \\
\end{array}$ & $\begin{array}{l}19.3 \% \\
(0.07) \\
\end{array}$ & $\begin{array}{l}25.3 \% \\
(0.09) \\
\end{array}$ & $\begin{array}{l}11.4 \% \\
(0.07) \\
\end{array}$ & $\begin{array}{l}29.4 \% \\
(0.11) \\
\end{array}$ & $\begin{array}{l}10.4 \% \\
(0.07) \\
\end{array}$ & $\begin{array}{l}33.1 \% \\
(0.11) \\
\end{array}$ & $\begin{array}{l}16.1 \% \\
(0.07) \\
\end{array}$ & $\begin{array}{l}37.9 \% \\
(0.13) \\
\end{array}$ & $\begin{array}{l}21.5 \% \\
(0.08) \\
\end{array}$ \\
\hline Paraguay & $6.5 \%$ & $10.5 \%$ & $\begin{array}{l}1.5 \% \\
(0.1) \\
\end{array}$ & $\begin{array}{l}13.4 \% \\
(0.09) \\
\end{array}$ & $\begin{array}{c}-11.1 \% \\
(0.14) \\
\end{array}$ & $\begin{array}{l}49.2 \% \\
(0.23) \\
\end{array}$ & $\begin{array}{l}-4.5 \% \\
(0.17) \\
\end{array}$ & $\begin{array}{l}41.5 \% \\
(0.28) \\
\end{array}$ & $\begin{array}{c}8.3 \% \\
(0.19) \\
\end{array}$ & $\begin{array}{l}39.5 \% \\
(0.27) \\
\end{array}$ & $\begin{array}{l}2.5 \% \\
(0.2) \\
\end{array}$ & $\begin{array}{l}37.3 \% \\
(0.33) \\
\end{array}$ & $\begin{array}{l}11.6 \% \\
(0.25) \\
\end{array}$ & $\begin{array}{c}41.0 \% \\
(0.3) \\
\end{array}$ & $\begin{array}{l}15.0 \% \\
(0.28) \\
\end{array}$ & $\begin{array}{c}-11.9 \% \\
(0.28) \\
\end{array}$ & $\begin{array}{l}92.1 \% \\
(0.29) \\
\end{array}$ & $\begin{array}{l}16.6 \% \\
(0.25) \\
\end{array}$ & $\begin{array}{c}-21.1 \% \\
(0.03) \\
\end{array}$ & $\begin{array}{l}40.9 \% \\
(0.23) \\
\end{array}$ \\
\hline Peru & $8.9 \%$ & $4.7 \%$ & $\begin{array}{c}6.9 \% \\
(0.04) \\
\end{array}$ & $\begin{array}{c}2.4 \% \\
(0.03) \\
\end{array}$ & $\begin{array}{l}12.8 \% \\
(0.09) \\
\end{array}$ & $\begin{array}{c}8.8 \% \\
(0.05) \\
\end{array}$ & $\begin{array}{l}12.5 \% \\
(0.09)\end{array}$ & $\begin{array}{l}18.5 \% \\
(0.06) \\
\end{array}$ & $\begin{array}{c}10.5 \% \\
(0.1) \\
\end{array}$ & $\begin{array}{l}18.9 \% \\
(0.06)\end{array}$ & $\begin{array}{l}2.2 \% \\
(0.1) \\
\end{array}$ & $\begin{array}{l}18.7 \% \\
(0.07) \\
\end{array}$ & $\begin{array}{c}7.0 \% \\
(0.12) \\
\end{array}$ & $\begin{array}{l}17.3 \% \\
(0.07) \\
\end{array}$ & $\begin{array}{c}-2.5 \% \\
(0.1) \\
\end{array}$ & $\begin{array}{l}16.0 \% \\
(0.07)\end{array}$ & $\begin{array}{l}15.9 \% \\
(0.14) \\
\end{array}$ & $\begin{array}{c}43.2 \% \\
(0.1) \\
\end{array}$ & $\begin{array}{l}24.2 \% \\
(0.18) \\
\end{array}$ & $\begin{array}{l}42.4 \% \\
(0.13) \\
\end{array}$ \\
\hline Uruguay & $44.0 \%$ & $16.9 \%$ & $\begin{array}{l}38.7 \% \\
(0.05) \\
\end{array}$ & $\begin{array}{l}19.0 \% \\
(0.04) \\
\end{array}$ & $\begin{array}{l}38.2 \% \\
(0.07) \\
\end{array}$ & $\begin{array}{l}17.1 \% \\
(0.05) \\
\end{array}$ & $\begin{array}{l}44.6 \% \\
(0.08) \\
\end{array}$ & $\begin{array}{l}-9.0 \% \\
(0.06) \\
\end{array}$ & $\begin{array}{l}41.9 \% \\
(0.08) \\
\end{array}$ & $\begin{array}{l}-6.5 \% \\
(0.06) \\
\end{array}$ & $\begin{array}{l}40.9 \% \\
(0.09) \\
\end{array}$ & $\begin{array}{l}-7.2 \% \\
(0.06) \\
\end{array}$ & $\begin{array}{c}44.6 \% \\
(0.1) \\
\end{array}$ & $\begin{array}{l}-6.0 \% \\
(0.07) \\
\end{array}$ & $\begin{array}{c}46.5 \% \\
(0.1) \\
\end{array}$ & $\begin{array}{l}-0.1 \% \\
(0.09) \\
\end{array}$ & $\begin{array}{l}62.1 \% \\
(0.12) \\
\end{array}$ & $\begin{array}{c}2.3 \% \\
(0.09) \\
\end{array}$ & $\begin{array}{l}62.5 \% \\
(0.13) \\
\end{array}$ & $\begin{array}{l}12.5 \% \\
(0.11) \\
\end{array}$ \\
\hline $\begin{array}{l}\text { Latin America } \\
\text { (13 countries) }\end{array}$ & $21.4 \%$ & $-4.5 \%$ & $\begin{array}{l}13.9 \% \\
(0.02) \\
\end{array}$ & $\begin{array}{l}-4.3 \% \\
(0.02) \\
\end{array}$ & $\begin{array}{l}14.4 \% \\
(0.03) \\
\end{array}$ & $\begin{array}{l}3.0 \% \\
(0.03) \\
\end{array}$ & $\begin{array}{l}25.7 \% \\
(0.03) \\
\end{array}$ & $\begin{array}{l}12.2 \% \\
(0.03) \\
\end{array}$ & $\begin{array}{l}24.9 \% \\
(0.04) \\
\end{array}$ & $\begin{array}{l}12.2 \% \\
(0.03) \\
\end{array}$ & $\begin{array}{l}22.5 \% \\
(0.04) \\
\end{array}$ & $\begin{array}{l}12.2 \% \\
(0.04) \\
\end{array}$ & $\begin{array}{l}23.1 \% \\
(0.04) \\
\end{array}$ & $\begin{array}{l}11.2 \% \\
(0.04) \\
\end{array}$ & $\begin{array}{l}23.6 \% \\
(0.04) \\
\end{array}$ & $\begin{array}{l}10.5 \% \\
(0.04) \\
\end{array}$ & $\begin{array}{l}32.4 \% \\
(0.05) \\
\end{array}$ & $\begin{array}{l}18.8 \% \\
(0.04) \\
\end{array}$ & $\begin{array}{l}31.7 \% \\
(0.05) \\
\end{array}$ & $\begin{array}{l}18.8 \% \\
(0.05) \\
\end{array}$ \\
\hline
\end{tabular}


The drops in unexplained earnings gaps can arise either as a result of a general trend of gaps decreasing in the segments considered of the labor markets (that is, those for professionals and technicians, where teachers are involved), or can also be the result of changes over time of the distribution of individuals' observable characteristics. To further explore the effects of labor market trends versus changes in labor markets composition, Table 5 presents a "matching after matching" exercise (Ñopo and Hoyos, 2010) disentangling both. The first stage of matching is performed with the full set of observable characteristics, matching teachers with other professionals and technicians in both moments under consideration (circa 1997 and circa 2007), as it has already been performed. After that, the matching after matching exercise is performed for the two matched sets of workers, matching the circa 1997 data with the one from circa 2007. In this way not only teachers and non-teachers show no differences in observable characteristics, but also they show no changes in the distribution of those characteristics during the period under analysis. The results, shown in Table 5, indicate that there is more evidence of a general trend of decreasing gaps than one of an improvement of teachers' characteristics. The change in earnings gaps due to changes in the distributions of observable characteristics is positive and higher for preschool and elementary teachers, but compensated by the change in the counterfactual component.

Table 5. Decomposition of the Change in Unexplained Earnings Gap circa 2007- circa 1997 (after Controlling by the Full Set of Observable Characteristics)

\begin{tabular}{lccc}
\hline \hline & $\begin{array}{c}\text { Counterfactual } \\
\text { Change if no } \\
\text { Change in X's }\end{array}$ & $\begin{array}{c}\text { Part of the } \\
\text { Change due to } \\
\text { Change in X's }\end{array}$ & Total Change \\
\hline Pre-school and Elementary Teachers vis-à-vis & $-65.3 \%$ & $11.2 \%$ & $-54.1 \%$ \\
Other Professionals and Technicians & $(0.04)$ & $(0.00)$ & $(0.00)$ \\
High School Teachers vis-à-vis Other & $-22.0 \%$ & $9.0 \%$ & $-13.0 \%$ \\
Professionals and Technicians & $(0.07)$ & $(0.00)$ & $(0.00)$ \\
\hline \hline
\end{tabular}

Source: Authors' calculations based on household surveys.

\subsection{Changes in the Distribution of the Unexplained Earnings Gap}

After matching individuals on the basis of observable characteristics it is possible to explore not only the average but also the distribution of the earnings gaps in each period. In general, as reported in Mizala and Ñopo (2011), pre-school and elementary school teachers' underpayment is more pronounced among older and more educated workers, household heads, part-time workers, and those who report having more than one job. As reported in Table 5, during the period under analysis there is evidence of a general trend over all the labor markets for a reduction of earnings gaps. Within this matching exercise, nonetheless, it is possible to explore the segments of the 
labor markets for which the drops in gaps have been more pronounced. The bigger drops in earnings gaps for pre-school and elementary teachers occurred among younger individuals, those with higher education (secondary complete or more), with no elders at home, part-time workers and those with no secondary jobs. For high school teachers, the earnings gaps are more pronounced among household heads and those holding more than one job. Among these teachers there is no particular segment of the market for which the gap dropped particularly more than the rest. $^{4}$

Figure 2 shows the unexplained earnings gaps along the individuals' earnings distribution. The first panel of it shows the unexplained earnings gap that pre-school and elementary school face in comparison to other professionals and technicians; the second panel does it for high school teachers. Both show that the earnings gap between teachers and comparable workers in Latin America are driven by pay differences at the top percentiles of the earnings distribution. In this regard there is no much cross-country heterogeneity. ${ }^{5}$

This first panel of Figure 2 evidences that, after controlling by the full set of observable characteristics, there are no major differences in hourly earnings between pre-school and elementary teachers and other professional and technicians for the bottom third of the population circa 2007. The second panel depicts similar results, with smaller changes between circa 1997 and 2007.

Figure 2. Unexplained Earnings Gaps along Percentiles of the Earnings Distribution (after Controlling by the Full set of Observable Characteristics)

a. Pre-School and Elementary Teachers vs. Other Professionals and Technicians

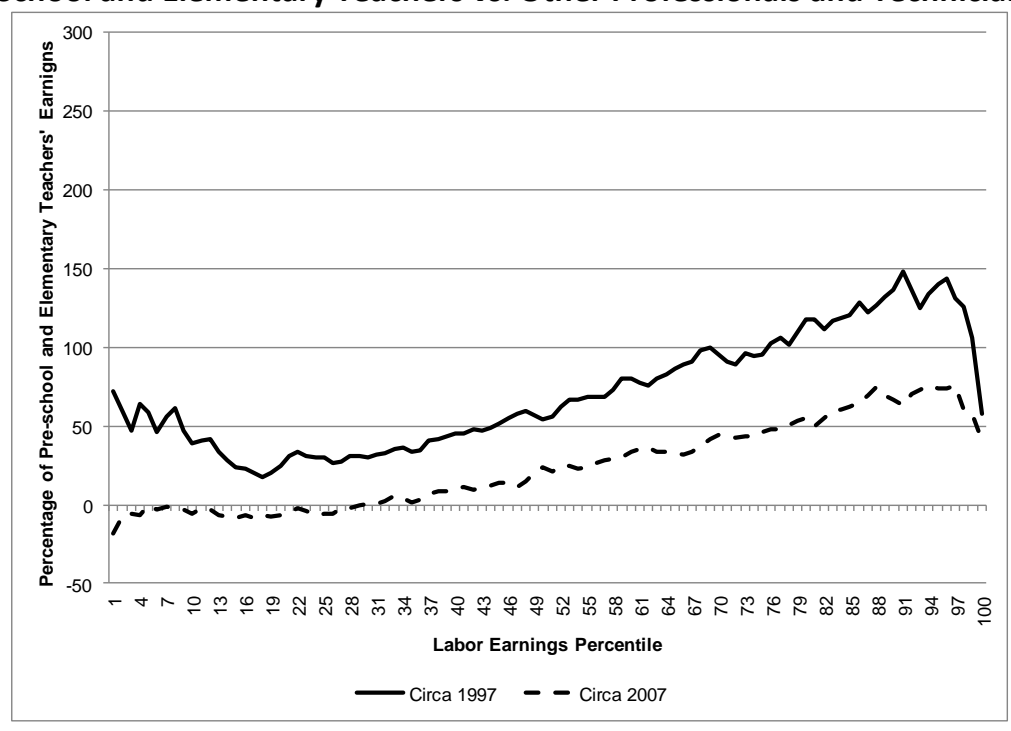

\footnotetext{
${ }^{4}$ Confidence intervals for the unexplained earnings gap between teachers and non-teachers by different characteristics, after controlling by the full set, can be found on Figures A1 and A2 of the Appendix.

${ }^{5}$ Country-by-country results of the unexplained earnings gaps along percentiles of the earnings distribution are not shown here but these are available from the authors upon request.
} 


\section{b. High School Teachers vs. Other Professionals and Technicians}

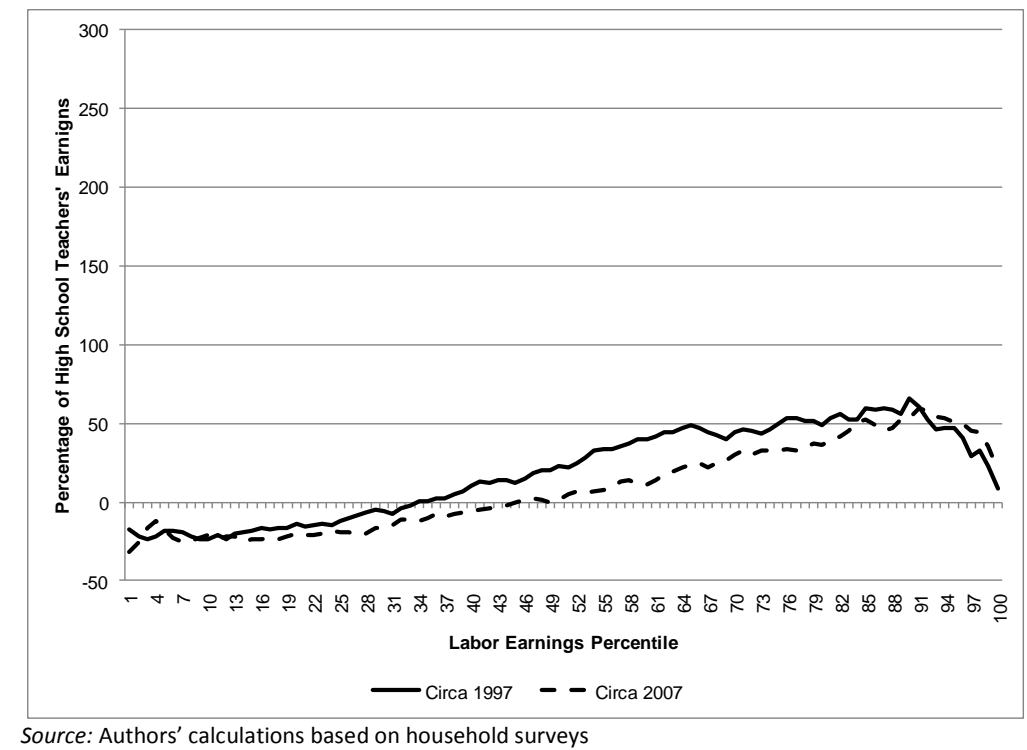

\subsection{Exploring the Role of Amenities: Schedules, Vacations, Secondary Jobs and Tenure}

"Typical policy discussions about the choice of a teaching career highlight the extra amenities that come with a teaching job. Two of the most salient of those amenities are the shorter (and flexible) job schedules, on the one hand, and the more stability that the profession enjoys, on the other. As it is typical in economics, these extra amenities have to come at a price. In this case such price would be expressed in terms of earnings disparities between teachers and their peers." (Mizala and Ñopo, 2011). The shorter and flexible job schedules at the teaching profession imply, in turn, extra freedom for the possibility of holding a second job. As a matter of fact, in Table 2 we showed that while more than half of the teachers work part-time at their main job (30 hour per week or less) it is only around one-fifth of other professionals and technicians who do so. Not only that, but also the share of teachers who report having a secondary job is higher than that of other professionals and technicians, especially for those teaching at the high school level.

With these considerations we analyze the role of job schedules going beyond the measurement of earnings gaps in hourly terms. We explore earnings gaps in monthly and yearly earnings. These earnings are measured in terms of purchasing power parity (PPP, US\$ 2000) as well. Monthly earnings correspond to the monthly income received in the main occupation (i.e., the monthly value of hourly earnings). Yearly earnings try to capture the fact that job-breaks are not the same across occupations, but since information about vacation periods is not available in the household surveys we built a proxy. Yearly earnings are computed as follows: for teachers, we assume a twomonth paid vacation period so that teachers monthly earnings are multiplied by a 12/10 ratio; for other professionals and technicians dependent workers we assume a one-month paid vacation period so that monthly earnings are multiplied by a 12/11 ratio; and for independent workers we assume no paid vacations so that their monthly earnings are multiplied by $12 / 12=1$. 
Table 6 presents earnings gap decompositions for monthly and yearly earnings, for pre-school and elementary school teachers and for high school teachers (in both cases, vis-à-vis other professionals and technicians). First, provided teachers report working less hours per month (or per week) than their counterparts at their main occupation it comes at no surprise that that monthly earnings gaps are higher than the hourly eanrings gaps previously reported in Table 4, both at their original measure and at the one that remains after controlling for the full set of observable characteristics. Second, provided the proxy-adjustment of yearly earnings "inflate" teachers' earnings (with respect to that of other professionals and technicians), it also comes at no surprise that the measure of yearly earnings gaps is smaller than the one for monthly earnings gaps. The result arises simply by construction. Third, these proxied measures of yearly earnings gaps are also bigger than the hourly earnings gaps in Table 4. Furthermore, a measure of hourly earning gaps that adjusts by the differential vacations (as proxied on the yearly measures) delivers, by construction, earnings gaps that are smaller than the hourly earnings gaps reported in Table 4, and are still positive and statistically significant (this last result is not shown here but available upon request).

Table 6. Unexplained Earnings Gaps Controlling by the Full set of Observable Characteristics (by different measures of earnings at the main occupation)

\begin{tabular}{|c|c|c|c|c|c|c|c|c|}
\hline & \multicolumn{4}{|c|}{ Monthly earnings } & \multicolumn{4}{|c|}{ Yearly earnings } \\
\hline & \multicolumn{2}{|c|}{ Original gap } & \multicolumn{2}{|c|}{ Full set } & \multicolumn{2}{|c|}{ Original gap } & \multicolumn{2}{|c|}{ Full set } \\
\hline & C-97 & C-07 & C-97 & C-07 & C-97 & C-07 & C-97 & C-07 \\
\hline \multicolumn{9}{|c|}{ Pre-School and Elementary Teachers vis-à-vis Other Professionals and Technicians } \\
\hline Region average & $145.0 \%$ & $64.7 \%$ & $\begin{array}{l}96.1 \% \\
(0.03)\end{array}$ & $\begin{array}{l}58.8 \% \\
(0.02) \\
\end{array}$ & $119.3 \%$ & $47.5 \%$ & $\begin{array}{l}75.0 \% \\
(0.03) \\
\end{array}$ & $\begin{array}{l}42.0 \% \\
(0.02) \\
\end{array}$ \\
\hline \multicolumn{9}{|c|}{ High School Teachers vis-à-vis Other Professionals and Technicians } \\
\hline Region average & $61.8 \%$ & $25.4 \%$ & $\begin{array}{l}66.4 \% \\
(0.08) \\
\end{array}$ & $\begin{array}{l}47.9 \% \\
(0.06) \\
\end{array}$ & $44.8 \%$ & $12.3 \%$ & $\begin{array}{l}47.1 \% \\
(0.07) \\
\end{array}$ & $\begin{array}{l}32.4 \% \\
(0.05) \\
\end{array}$ \\
\hline
\end{tabular}

Source: Authors' calculations based on household surveys.

Note: Standard errors in parentheses.

Table A2 (in the Appendix) shows the results of Table 6 dissagregated by country. There it can be seen that the higher gaps for monthly and yearly earnings (vis-à-vis those measured in hourly terms) hold for most countries in the region. Chile and, to a lesser extent, Panama are interesting exceptions as in these countries the gaps in yearly terms are similar to those originally measured in hourly terms. This reflects that the number of hours worked per week by teachers is not so different than the number of hours worked by other professional and technicians. In these two countries this claimed amenity of shorter job schedules is not so prevalent, and this is specially the case for the most recent data circa 2007. For other countries, after controlling for part-time work, the gaps in yearly terms and the gaps in hourly terms reach similar values as well. 
Next, we incorporate into the analysis an extra possibility that teachers enjoy regarding their use of their time, the holding of a second job. As highlighted in Table 2, teachers' propensity to have a second job is higher than that of other professionals and technicians, especially for those teaching at the high-school level (for this later group almost one in four teachers have a second job). This expands the income generation possibilities for teachers and may also be considered as an amenity linked to the profession. So analyze next the earnings gaps in labor income for the main and secondary sources.

Many countries within our data report the existence of second jobs but only in six of them it is possible to obtain data for earnings, hours worked per week and type of activity in the second job: Bolivia, Brazil, Costa Rica, Ecuador, Nicaragua and Paraguay. The next part of the analysis will then focus on these countries. The sample drops dramatically as a result of two combined restrictions on the data. First, this sample of six countries approximately constitutes $75 \%$ of the observations that have been used for the previous results. Second, within the six countries we restrict attention to those individuals who hold a second job and have information on earnings, hours worked per week and type of activity in the second job. This implies restricting the sample to approximately $15 \%$ of workers in these 6 countries. Combining the two restrictions, the resulting sample represents around 12\% of the original teachers sample circa 1997 and circa 2007, 8\% of the original non-teachers sample circa 1997 and 6\% circa 2007. Brazil becomes utterly representative, holding around $85 \%$ of the observations in both periods. Also, the share of household heads and (as expected) part-time workers is higher in this sub-sample in comparison to the original sample. ${ }^{6}$

Table 7 shows selected descriptive statistics for the sub-sample. The upper panel of the table (main job) depicts two important patterns that are in line with what was previously reported in Table 3. First, part-time workers at their main job earn more than those who are not, and a greater share of teachers report working part time. Second, to an important extent the drops in earnings gap at the main job are due to a drop in relative earnings for other professional and technicians. The intermediate panel of the table shows data from the second job. Two results emerge. First, to an important extent (with the exception of high school teachers circa 2007) the second job of teachers tends to be at another teaching position. Second, earnings gaps at second jobs did not change as much as they did for main jobs, and this is mainly a consequence of the relative improvement of other professionals and technicians' earnings during the period. The bottom panel of Table 7 shows descriptive statistics for main and secondary jobs combined (i.e., earnings are equal to the sum of main job and second job monthly earnings). The evidence still points towards a higher number of working hours, and higher earnings, for non-teachers than for teachers.

\footnotetext{
${ }^{6}$ These results are not reported here but available upon request.
} 
Table 7. Descriptive Statistics

( 6 countries with data on second job)

\begin{tabular}{|c|c|c|c|c|c|c|}
\hline \multicolumn{7}{|c|}{ Sub-sample of workers that reported having a secondary job, the related activity, earnings and hours worked per week in this second job } \\
\hline & \multicolumn{2}{|c|}{ Pre-School and Elementary } & \multicolumn{2}{|c|}{ High School Teachers } & \multicolumn{2}{|c|}{ Other Professionals and } \\
\hline & Circa 1997 & Circa 2007 & Circa 1997 & Circa 2007 & Circa 1997 & Circa 2007 \\
\hline \multicolumn{7}{|c|}{ Main Job } \\
\hline \multicolumn{7}{|l|}{ Part-time work } \\
\hline Region Average & $76.9 \%$ & $76.9 \%$ & $58.6 \%$ & $65.5 \%$ & $50.6 \%$ & $43.5 \%$ \\
\hline \multicolumn{7}{|c|}{ Average hourly earnings (part-time workers)* } \\
\hline Region Average & 90.3 & 94.9 & 127.3 & 120.3 & 259.3 & 193.8 \\
\hline \multicolumn{7}{|c|}{ Average hourly earnings (non part-time workers)* } \\
\hline Region Average & 74.5 & 74.9 & 112.3 & 93.1 & 187.2 & 158.5 \\
\hline \multicolumn{7}{|c|}{ Second Job } \\
\hline \multicolumn{7}{|c|}{ Second job involves school-teaching activities } \\
\hline Region Average & $64.3 \%$ & $67.3 \%$ & $61.5 \%$ & $33.6 \%$ & $11.8 \%$ & $1.8 \%$ \\
\hline \multicolumn{7}{|c|}{ Average hourly earnings in second job* } \\
\hline Region Average & 98.2 & 107.3 & 179.0 & 140.3 & 233.8 & 278.8 \\
\hline \multicolumn{7}{|c|}{ Main and Second Jobs (combined) } \\
\hline Average hours worked pe & & & & & & \\
\hline Region Average & 46.2 & 46.7 & 47.7 & 48.3 & 53.3 & 52.8 \\
\hline \multicolumn{7}{|c|}{ Works over-time (50 hours a week or more) } \\
\hline Region Average & $41.5 \%$ & $40.8 \%$ & $48.0 \%$ & $49.6 \%$ & $64.0 \%$ & $64.6 \%$ \\
\hline \multicolumn{7}{|c|}{ Average monthly earnings in main and second jobs** } \\
\hline Region Average & 86.2 & 157.3 & 138.9 & 203.4 & 254.3 & 353.2 \\
\hline Observations & 581 & 686 & 235 & 394 & 1113 & 2084 \\
\hline Expanded Observations & 242672 & 305354 & 85904 & 147614 & 413246 & 812217 \\
\hline
\end{tabular}

Source: Authors' calculations based on household surveys.

* Average school teacher earnings in main job circa 1997 in each Country=100

** Average school teacher monthly earnings in main and second jobs (combined) circa 1997 in each Country=100

Table 8 shows the original and the unexplained earnings gap for main and secondary job (using hourly earnings), and the combination of both (using monthly earnings). Since we are restricting the sample to those workers that report having a second job, the "Full Set" specification does not include the "more than on job" variable. Additionally, we add another control variable: whether the worker's second job is related to school teaching or not after controlling by the full set of observable characteristics. The unexplained hourly earnings gaps at the second job are also positive but smaller than those at the main job for both periods. Both, the gaps at the main and second jobs decreased during the period but the drops are not statistically significant (perhaps due to the dramatic reduction in the sample size for this exercise with six countries and second job holders). The gaps in monthly earnings combining the two sources of income are not statistically different than the gaps in monthly earnings solely measured at the main job in Table 6 . For this latter there is also evidence that the gap decreased but this is not statistically significant.

Adding the control for teaching activities at the second job does not change much the unexplained earnings gaps. Teachers in their second jobs, being those involved or not with teaching duties, face earnings gaps vis-à-vis other professionals and technicians. This may reflect the existence of some 
individuals' unobservable characteristics (or abilities) that the labor markets reward for which teachers fare worse than their peers. This last result may call for some action rewarding selection policies for high ability individuals into the teaching profession. 
Table 8. Unexplained Earnings Gap Controlling by the Full set of Observable Characteristics and Teaching in the Second Job ( 6 countries with data on second job)

\begin{tabular}{|c|c|c|c|c|c|c|c|c|c|c|c|c|c|c|c|}
\hline & \multicolumn{9}{|c|}{ Hourly Earnings } & \multirow{2}{*}{\multicolumn{6}{|c|}{$\begin{array}{c}\text { Full Monthly earnings } \\
\text { Main and Second Job Combined }\end{array}$}} \\
\hline & \multicolumn{4}{|c|}{ Main Job } & \multicolumn{5}{|c|}{ Second Job } & & & & & & \\
\hline & \multicolumn{2}{|c|}{ Original gap } & \multicolumn{2}{|c|}{ Full set* } & Original gap & \multicolumn{2}{|c|}{ Full set* } & \multicolumn{2}{|c|}{$\begin{array}{l}\text { + Second job: } \\
\text { school teacher }\end{array}$} & \multicolumn{2}{|c|}{ Original gap } & \multicolumn{2}{|c|}{ Full set* } & \multicolumn{2}{|c|}{$\begin{array}{l}\text { +Second job: } \\
\text { school teacher }\end{array}$} \\
\hline & C-97 & C-07 & C-97 & C-07 & C-97 $\quad$ C-07 & C-97 & C-07 & C-97 & C-07 & C-97 & C-07 & C-97 & C-07 & C-97 & C-07 \\
\hline \multicolumn{16}{|c|}{ Pre-School and Elementary Teachers vis-à-vis Other Professionals and Technicians } \\
\hline Region average & $187.0 \%$ & $104.4 \%$ & $\begin{array}{c}120.3 \% \\
(0.1) \\
\end{array}$ & $\begin{array}{l}97.2 \% \\
(0.11) \\
\end{array}$ & $138.2 \% 159.6 \%$ & $\begin{array}{c}93.2 \% \\
(0.1) \\
\end{array}$ & $\begin{array}{l}79.9 \% \\
(0.08) \\
\end{array}$ & $\begin{array}{c}70.2 \% \\
(0.2) \\
\end{array}$ & $\begin{array}{l}72.3 \% \\
(0.19) \\
\end{array}$ & $195.0 \%$ & $125.0 \%$ & $\begin{array}{l}99.7 \% \\
(0.09)\end{array}$ & $\begin{array}{l}85.6 \% \\
(0.06) \\
\end{array}$ & $\begin{array}{l}81.3 \% \\
(0.18) \\
\end{array}$ & $\begin{array}{l}92.2 \% \\
(0.14) \\
\end{array}$ \\
\hline \multicolumn{16}{|c|}{ High School Teachers vis-à-vis Other Professionals and Technicians } \\
\hline Region average & $103.7 \%$ & $61.1 \%$ & $\begin{array}{l}97.9 \% \\
(0.21)\end{array}$ & $\begin{array}{l}74.8 \% \\
(0.14)\end{array}$ & $30.7 \% \quad 98.7 \%$ & $\begin{array}{l}57.0 \% \\
(0.19)\end{array}$ & $\begin{array}{c}54.3 \% \\
(0.2)\end{array}$ & $\begin{array}{l}13.3 \% \\
(0.37)\end{array}$ & $\begin{array}{c}57.5 \% \\
(0.3)\end{array}$ & $83.0 \%$ & $73.6 \%$ & $\begin{array}{l}87.9 \% \\
(0.19)\end{array}$ & $\begin{array}{l}64.2 \% \\
(0.12)\end{array}$ & $\begin{array}{l}73.3 \% \\
(0.35)\end{array}$ & $\begin{array}{l}65.0 \% \\
(0.16)\end{array}$ \\
\hline
\end{tabular}

Source: Authors' calculations based on household surveys.

Standard errors in parentheses

* The Full Set specification does not include the variable "more than one job" as we are restricting our comparison to those who report having a second job. 
The other amenity we explore in this sub-section is tenure. It has been typically claimed that the teaching profession entails more job stability than others. This may in turn convert into a compensating differential that teachers are willing to accept in the form of lower salaries. Next we assess the role of job tenure on the earnings gaps. Job tenure is defined here as the approximate number of years an individual has remained in the same job at the moment of the survey. As in the previous case with second jobs, this analysis cannot be performed for the thirteen countries of the original analysis. Data on job tenure is available in seven countries: Bolivia, Brazil, Honduras, Nicaragua, Panama, Paraguay and Uruguay. Restricting the data to these countries implies using $77 \%$ of the original data for teachers circa 1997 and $76 \%$ circa 2007 ; as well as $62 \%$ and $74 \%$ of the non-teachers' group for circa 1997 and circa 2007 respectively. No descriptive statistic within this restricted data set is significantly different than those reported in table 2 for the set of thirteen countries.

Figure 3 presents Kernel density estimations of job tenure for teachers and other professionals and technicians for the two years under analysis. It can be highlighted from that figure that, in fact, teachers enjoy a positive tenure gap vis-à-vis other professional and technicians. Additionally, such gap became more pronounced circa 2007. Both teaching groups increased their average tenure (by 1.7 years for pre-school and elementary teachers and by 1.5 years for school teachers) but the non-teaching group decreased it (by 0.6 years). Such widening of the tenure gap goes in line with the fact that, as shown in table 2, teachers became older during this period. As a matter of fact, average age increased, on the one hand, by 4.4 years for pre-school and elementary teachers and by 3.3 years for high school teachers; and on the other hand it only increased by 0.3 years for other professionals and technicians. All these inter-temporal average changes are statistically significant at the $1 \%$ level.

Figure 4 presents the non-parametric regressions of tenure on earnings. The figure shows that other professional and technicians earn more than teachers in both periods and such differences appear to increase with tenure. These results hold for every country considered. Nevertheless, this analysis does not take into account the role of observable characteristics. For that reason, we perform a matching exercise that controls for differences in observable characteristics next.

Table 9 shows the earnings gaps decompositions for pre-school and elementary school teachers and for high school teachers (vis-à-vis other professionals and technicians) using the current data subset, adding job tenure as a control variable. Adding job tenure as a control variable reduces the earnings gap for preschool and elementary teachers in both periods and for all specifications. These results give credence to the idea that job stability acts as a compensating differential. For high school teachers the gap declines after adding job tenure as a control circa 2007; but circa 1997 it increases. These results suggest that job stability has higher intrinsic value nowadays. Table A3 (Appendix) depicts similar results by country. These results, however, must be taken with caution due to the smaller sample size and (especially due to) the smaller size of the common support. 
Figure 3. Estimated Kernel Distributions of Job Tenure

(7 countries with data on job tenure)

a. Pre-School and Elementary Teachers vs. Other Professionals and Technicians
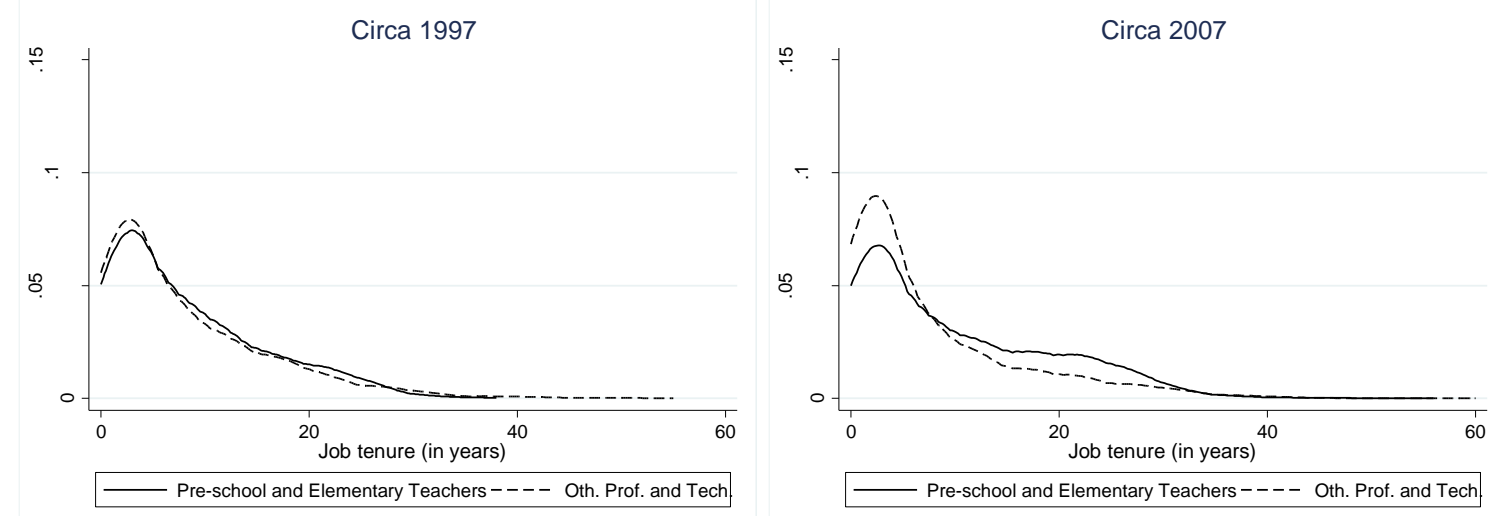

b. High School Teachers vs. Other Professionals and Technicians
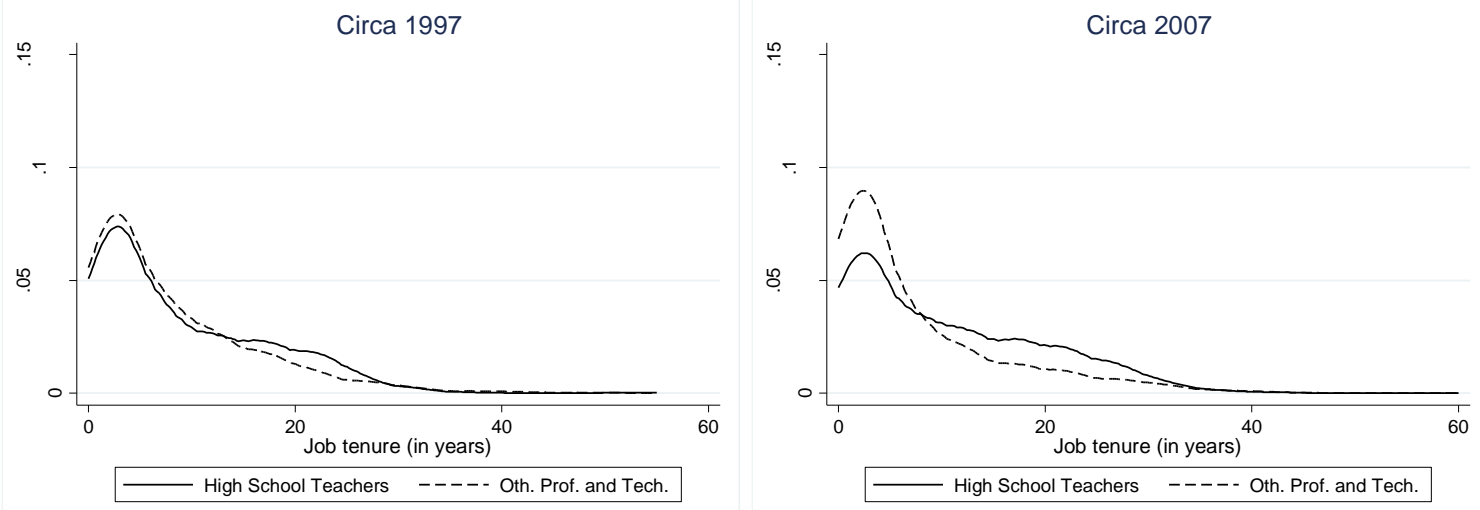

Source: Authors' calculations based on household surveys Bandwidth: 2

Figure 4. Estimated Kernel Regression Functions: Hourly Earnings vs. Job Tenure (7 countries with data on job tenure)

a. Pre-School and Elementary Teachers vs. Other Professionals and Technicians 

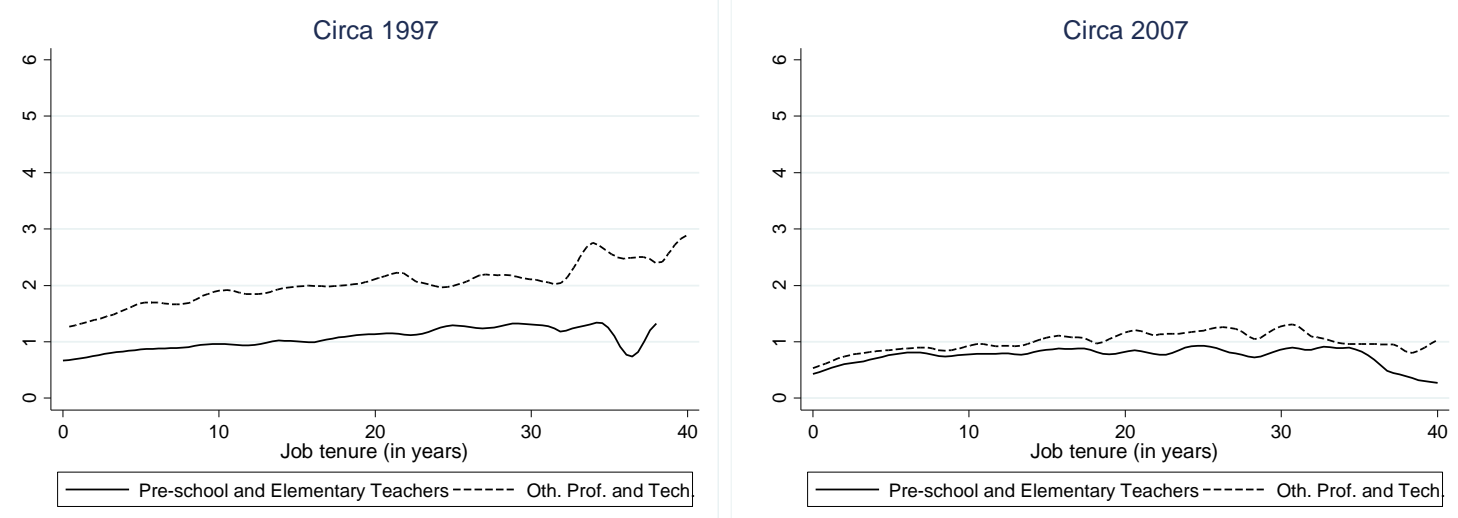

\section{b. High School Teachers vs. Other Professionals and Technicians}
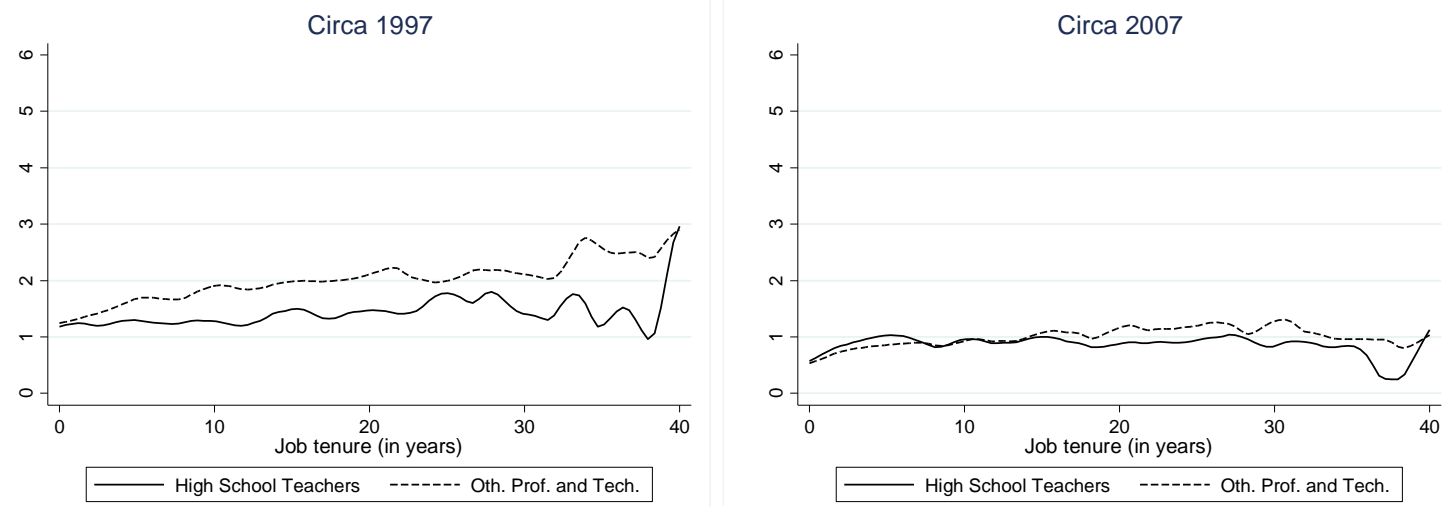

Source: Authors' calculations based on household surveys Bandwidth: 2 
Table 9. Unexplained Earnings Gaps after Controlling by the Full set of Observable Characteristics and Job Tenure (7 countries with data on job tenure)

\begin{tabular}{|c|c|c|c|c|c|c|c|c|c|c|c|c|c|c|c|c|c|c|}
\hline & \multicolumn{6}{|c|}{ Hourly earnings } & \multicolumn{6}{|c|}{ Monthly earnings } & \multicolumn{6}{|c|}{ Yearly earnings } \\
\hline & \multicolumn{2}{|c|}{ Original gap } & \multicolumn{2}{|c|}{ Full set } & \multicolumn{2}{|c|}{ + Tenure } & \multicolumn{2}{|c|}{ Original gap } & \multicolumn{2}{|c|}{ Full set } & \multicolumn{2}{|c|}{ + Tenure } & \multicolumn{2}{|c|}{ Original gap } & \multicolumn{2}{|c|}{ Full set } & \multicolumn{2}{|c|}{+ Tenure } \\
\hline & C-97 & C-07 & C-97 & C-07 & C-97 & C-07 & C-97 & C-07 & C-97 & C-07 & C-97 & C-07 & C-97 & C-07 & C-97 & C-07 & C-97 & C-07 \\
\hline \multicolumn{19}{|c|}{ Pre-School and Elementary Teachers vis-à-vis Other Professionals and Technicians } \\
\hline Region average & $104.4 \%$ & $24.7 \%$ & $\begin{array}{l}95.8 \% \\
(0.02)\end{array}$ & $\begin{array}{l}37.3 \% \\
(0.02) \\
\end{array}$ & $\begin{array}{l}87.6 \% \\
(0.05) \\
\end{array}$ & $\begin{array}{l}33.0 \% \\
(0.03) \\
\end{array}$ & $177.9 \%$ & $66.1 \%$ & $\begin{array}{c}108.6 \% \\
(0.03)\end{array}$ & $\begin{array}{l}43.3 \% \\
(0.02) \\
\end{array}$ & $\begin{array}{c}103.6 \% \\
(0.06)\end{array}$ & $\begin{array}{l}43.0 \% \\
(0.04) \\
\end{array}$ & $148.3 \%$ & $48.6 \%$ & $\begin{array}{l}86.0 \% \\
(0.03) \\
\end{array}$ & $\begin{array}{l}28.0 \% \\
(0.02) \\
\end{array}$ & $\begin{array}{l}81.7 \% \\
(0.05) \\
\end{array}$ & $\begin{array}{l}28.9 \% \\
(0.04) \\
\end{array}$ \\
\hline \multicolumn{19}{|c|}{ High School Teachers vis-à-vis Other Professionals and Technicians } \\
\hline Region average & $25.6 \%$ & $-2.7 \%$ & $\begin{array}{l}29.1 \% \\
(0.06)\end{array}$ & $\begin{array}{l}16.7 \% \\
(0.06)\end{array}$ & $\begin{array}{l}45.6 \% \\
(0.09)\end{array}$ & $\begin{array}{l}13.0 \% \\
(0.07)\end{array}$ & $64.3 \%$ & $20.9 \%$ & $\begin{array}{l}45.7 \% \\
(0.08)\end{array}$ & $\begin{array}{l}22.8 \% \\
(0.07)\end{array}$ & $\begin{array}{l}53.2 \% \\
(0.11)\end{array}$ & $\begin{array}{l}18.7 \% \\
(0.09)\end{array}$ & $46.7 \%$ & $8.1 \%$ & $\begin{array}{l}29.7 \% \\
(0.07)\end{array}$ & $\begin{array}{c}9.8 \% \\
(0.06)\end{array}$ & $\begin{array}{c}38.2 \% \\
(0.1)\end{array}$ & $\begin{array}{c}6.8 \% \\
(0.08)\end{array}$ \\
\hline
\end{tabular}

Source: Authors' calculations based on household surveys.

Note: Standard errors in parentheses. 


\section{Conclusions}

This paper examines whether teachers' earnings in Latin America are similar to those of other professionals and technicians, and how these earnings gaps evolved between circa 1997 and circa 2007. Since the available empirical evidence has shown that the sign and magnitude of the conditional earnings differential between teachers and other workers depend crucially on the definition of the comparison group, we build upon the results of Ñopo and Mizala (2011) using the methodology developed in Ñopo (2008). This approach emphasizes earnings differences in the supports of the distributions of observable characteristics and provides insights into the distribution of unexplained pay differences, which nurtures our comparison between periods. Furthermore, using the matching after matching approach we were able to provide further insights on the change of the earnings gap during the decade under analysis.

The results show that teachers are underpaid vis-à-vis other professionals and technicians in Latin America in both periods: circa 1997 and circa 2007; however, these gaps decreased. Nonetheless, there is an important cross-country heterogeneity behind the region averages. In particular, Brazil affects greatly the region averages due to its size. Despite this, the main conclusions hold if we include Brazil or not: High school teachers are more educated than other professionals and technicians but their years of education are not properly rewarded in the labor market. Working part-time is a characteristic that explains teachers' underpayment -pre-school and elementary school teachers enjoy greater flexibility in the work; moreover, job tenure and job schedules have an important role in explaining the earnings gap: the first is positively related with the earnings gap, and the second states that not considering job-brake periods (vacations) may lead to overestimate the earnings gap. Teachers' lower earnings are being compensated through lower effective labor in the main job, which not only eases prospects of having a family but also eases the decision of having/finding a second job, accepting the possibility of lower (main job) earnings.

Although being able to work part-time is a major reason behind the decision of having a second job, is important to explore further this possibility; in fact, a greater share of teachers report having a second job. We find that teachers are also being underpaid in their second job vis-à-vis other professionals and technicians, although these differences are smaller than in their main job, and decreased throughout the decade. Even though these results suggest that having a second job acts as a compensating differential, the data set for this analysis is very small and will be hard to conclude in this respect and other results obtained.

In general we found that the earnings gap decreased for each of the segments of the population provided by the control variables. Even more interesting, preschool and elementary teachers' earnings gap decreased importantly during the decade, especially for those who are females, younger and work part-time. Furthermore, in the hypothetical situation of no changes over time in the distribution of characteristics, results suggest an important decrease in the earnings gaps driven by the unexplained component of the gap, particularly for pre-school and primary teachers. All in all, the analysis performed provides evidence that the wage gap decreased during the ten 
year span of analysis, driven by the change in preschool and primary teachers' underpayment throughout the time span and a decrease in non-teachers real income.

Moreover, important differences along the earnings distribution were found. Teachers in the highest percentiles of the earnings distribution earn less than other professionals and technicians, however, these earnings differences decreased throughout the decade. At the same time, teachers in the bottom percentiles tend to have similar or higher earnings than comparable workers. This can be explained because in many countries teachers are rewarded trough a single salary schedule which implies a salary structure much more compressed than the one of other professionals and technicians.

This salary structure is appealing to teachers as a fair way to compensate everyone, because it does not make distinctions that might disturb relationships among them. In this scheme equal pay is provided regardless of differing efforts and abilities; salaries are unrelated to the activities performed at the schools; being seniority and, to a lesser extent, the level of education the primary basis for any pay increase, which means that in the teaching profession loyalty rather than actual job performance is rewarded.

This system implies that the teaching profession probably attracts people with a preference for job stability and security, and at the same time equal pay regardless of performance penalizes the highly effective teachers who should be earning more. Thus, the single salary schedule might not be attractive to high performing teachers. For this reason, several countries are reforming traditional systems of recruiting teachers as well as mechanisms of paying and rewarding them, in order to attract and retain highly qualified individuals into teaching, and to get teachers to work hard to raise student learning (OECD, 2009). 


\section{References}

Allegretto, S., Corcoran, S., and Mishel, L. (2004). How does teacher pay compare?. Methodological challenges and answers. Economic Policy Institute.

Allegretto, S., Corcoran, S., and Mishel, L. (2008). "Teaching Penalty: Teacher Pay Losing Ground". Economic Policy Institute.

Asadullah, M. (2006). "Pay differences between teachers and other occupations: Some empirical evidence from Bangladesh". Journal of Asian Economics 17, 1044-1065.

Ballou, D. and Podgursky, M. (1997). "Recruiting smarter teachers". Journal of Human Resources, 30(2), 326-338.

Barber Michael and Mona Mourshed. 2007. "How the World's Best-Performing School Systems Come Out On Top", McKinsey \& Company, Social Sector Office. http://www.mckinsey.com/clientservice/socialsector/resources/pdf/Worlds School Syste ms Final.pdf

Clotfelter, T., H.F. Ladd y J.L.Vigdor, "Teacher credentials and student achievement: Longitudinal analysis with student fixed effects" Economics of Education Review 26(6), 2007.

Darling-Hammond, L. (2001). "El derecho de aprender. Crear buenas escuelas para todos". Barcelona: Ariel, 460p.

Figlio, D. (1997). “Teacher salaries and teacher quality”. Economics Letters, 55(2), 267-271.

Figlio, D. and L. Kenny, (2006), "Individual teacher incentives and student performance" NBER Working paper № 12627

Goldhaber, D. and Brewer, D. (1997). "Why don't schools and teachers seem to matter?: Assessing the impact of unobservables on educational productivity, Journal of Human Resources 32(3), 505-523.

Harris, D. and Adams, S. (2007). "Understanding the level and causes of teacher turnover: A comparison with other professions". Economics of Education Review 26(3), 325-337

Herrero, V., De Santis, M. and Gertler, H. (2003). "El ingreso de los docentes en la Argentina: es alto o bajo?". Mimeo Instituto de Economía y Finanzas Facultad de Ciencias Económicas, Universidad Nacional de Córdoba.

Heutel, G. (2009). "Testing implications of a tournament model of school district salary schedules". Economics of Education Review 28(1), 143-151.

Imazeki, J. (2005). "Teacher salaries and teacher attrition". Economics of Education Review 24(4), 431-449. 
Komenan, A.G. and C. Grootaert, "Pay differences between teachers and other occupations: Some empirical evidence from Côte D' Ivoire," Economics of Education Review, 9(3): 209-217, 1990.

Kukla-Acevedo, S., "Do teacher characteristics matter? New results on the effects of teacher preparation on student achievement," Economics of Education Review 28: 49-57, 2009.

Liang, X. (1999). "Teacher Pay in 12 Latin American Countries: How Does Teacher Pay Compare to Other Professions, What Determines Teacher Pay, and Who Are the Teachers?".Latin America and the Caribbean Region Human Development Department Paper 49. World Bank, Washington, D.C.

Limarino, W.H. (2005). "Are Teachers Well Paid in Latin America and the Caribbean?" Vegas, E. (ed.), Incentives to Improve Teaching. Lessons from Latin America", Directions in Development, World Bank. Ed., 3, 63: 102.

Loeb, S. and M. Page (2000). "Examining the link between wages and quality in the teachers workforce. The role of alternative labor market opportunities and non-pecuniary variation". Review of Economics and Statistics 82(3), 393-408.

López-Acevedo, G., and A. Salinas. 2000. "Teachers' Salaries and Professional Profile in Mexico". Latin America and the Caribbean Region, World Bank, Washington, D.C. Processed.

Mincer, J. 1974. "Schooling, Experience, and Earnings". New York: Columbia University Press.

Mizala, A. and Romaguera, P. (2005), "Teachers' Salary Structure and Incentives in Chile". Vegas, E. (ed.), Incentives to Improve Teaching. Lessons from Latin America", Directions in Development, World Bank.

Mizala, A. and Ñopo, H., (2011). "Teachers' Salaries in Latin America. How Much are They (under or over) Paid?". IZA Discussion Papers 5947, Institute for the Study of Labor (IZA).

Naper, L. (2010). "Teacher hiring practices and educational efficiency". Economics of Education Review 29(4), 658-668.

Ñopo, H., (2008). "Matching as a Tool to Decompose Wage Gap". Review of Economics and Statistics, 90(2), 290-299, 03.

Ñopo, H. and A. Hoyos (2010). "Evolution of Gender Wage Gaps in Latin America at the Turn of the Twentieth Century: An Addendum to "New Century, Old Disparities"," IZA Discussion Papers 5086, Institute for the Study of Labor (IZA).

OECD (2009). "Evaluating and Rewarding the Quality of Teachers International Practices". 
Ortega, D. (2010). "The effect of wage compression and alternative labor market opportunities on teacher quality in Venezuela". Economics of Education Review in Press, Corrected Proof, Available online 4 February 2010.

Piras, C., and Savedoff, B. (1998). "How much do teachers earn?". IDB Working, Paper 375. InterAmerican Development Bank, Washington, D.C.

Player, D. (2009). "Monetary returns to academic ability in the public teacher labor market". Economics of Education Review 28(2), 277-285.

Podgursky, M. and Tongrut, R. (2006). "(Mis-)Measuring the Relative Pay of Public School Teachers". Education Finance and Policy, 1(4), 425-440.

Psacharopoulos, G., Valenzuela, J. and Arends, G. (1996). "Teacher Salaries in Latin America: A Review". Economics of Education Review 15(4), 401-6.

Rivas, H. and Lavarreda, J. (2008). "Análisis de las remuneraciones de los docentes del sector público en Guatemala". Informe Final para el Ministerio de Educación de Guatemala.

Rivkin, S., Hanushek, E. and Kain, J. (2005). "Teachers, Schools, and Academic Achievement". Econometrica, Econometric Society 73(2), 417-458.

Rockoff, J. E. (2004). "The Impact of Individual Teachers on Student Achievement: Evidence from Panel Data", The American Economic Review 94(2), 247-252.

Saavedra, J. (2004). "La situación laboral de los maestros respecto de otros profesionales. Implicancias para el diseño de políticas salariales y de incentivos".

Scafidi, B., Sjoquist, D. and Stinebrickner, T. (2007). "Race, poverty, and teacher mobility". Economics of Education Review 26(2), 145-159.

Urquiola, M., Jimenez, W., Talavera, M. L. and W. Hernani (2000). Los Maestros en Bolivia: Impacto, Incentivos y Desempeño. Maestrías para el desarrollo. Universidad católica Boliviana.

Zymelman, M. and J. DeStephano, "Primary school teachers'salaries in sub-Saharan Africa", World Bank Discussion Papers N²4, 1989. 
Table A1. Occupational Codes Included in the Definition of Teachers and Comparison Groups

\begin{tabular}{|c|c|c|}
\hline \multirow{2}{*}{ Country } & \multicolumn{2}{|c|}{ Codes } \\
\hline & Circa 1997 & Circa 2007 \\
\hline \multicolumn{3}{|l|}{ Bolivia } \\
\hline Pre-School and Elementary Teachers & $334,335^{\star}$ & $233,331,332$ \\
\hline High School Teachers & $333^{\star}$ & 232 \\
\hline Other Professionals and Technicians & Major groups 1 and $2^{*}$ & Major groups 2 and 3 \\
\hline \multicolumn{3}{|l|}{ Brazil } \\
\hline Pre-School and Elementary Teachers & $214-217^{*}$ & $2311,2312,2313,3311^{\star \star}$ \\
\hline High School Teachers & $213,218^{\star}$ & $2321,3313^{\star \star}$ \\
\hline Other Professionals and Technicians & Major groups 1 and $2^{*}$ & Major groups 2 and $3^{\star \star}$ \\
\hline \multicolumn{3}{|l|}{ Chile } \\
\hline Pre-School and Elementary Teachers & $233,331,332$ & $233,331,332$ \\
\hline High School Teachers & 232 & 232 \\
\hline Other Professionals and Technicians & Major groups 2 and 3 & Major groups 2 and 3 \\
\hline \multicolumn{3}{|l|}{ Costa Rica } \\
\hline Pre-School and Elementary Teachers & $62,63^{\star}$ & $233,331,332$ \\
\hline High School Teachers & $61^{\star}$ & 232 \\
\hline Other Professionals and Technicians & Major group $0^{*}$ & Major groups 2 and 3 \\
\hline \multicolumn{3}{|l|}{ Dominican Republic } \\
\hline Pre-School and Elementary Teachers & $233,331,332$ & $233,331,332$ \\
\hline High School Teachers & 232 & 232 \\
\hline Other Professionals and Technicians & Major groups 1 and 2 & Major groups 2 and 3 \\
\hline \multicolumn{3}{|l|}{ Ecuador } \\
\hline Pre-School and Elementary Teachers & $233,331,332$ & $233,331,332$ \\
\hline High School Teachers & 232 & 232 \\
\hline Other Professionals and Technicians & Major groups 1 and 2 & Major groups 2 and 3 \\
\hline \multicolumn{3}{|l|}{ El Salvador } \\
\hline Pre-School and Elementary Teachers & $233,331,332$ & $233,331,332$ \\
\hline High School Teachers & 232 & 232 \\
\hline Other Professionals and Technicians & Major groups 2 and 3 & Major groups 2 and 3 \\
\hline \multicolumn{3}{|l|}{ Honduras } \\
\hline Pre-School and Elementary Teachers & $1249,1273^{\star \star \star}$ & $233,331,332$ \\
\hline High School Teachers & $1231^{\star \star \star}$ & 232 \\
\hline Other Professionals and Technicians & Major group 0 and $1^{\star \star \star}$ & Major groups 2 and 3 \\
\hline \multicolumn{3}{|l|}{ Nicaragua } \\
\hline Pre-School and Elementary Teachers & $233,331,332$ & $233,331,332$ \\
\hline High School Teachers & 232 & 232 \\
\hline Other Prof essionals and Technicians & Major groups 2 and 3 & Major groups 2 and 3 \\
\hline \multicolumn{3}{|l|}{ Panama } \\
\hline Pre-School and Elementary Teachers & $200-207^{*}$ & $291-297$ \\
\hline High School Teachers & $189-199^{\star}$ & $279-290$ \\
\hline Other Professionals and Technicians & Major group $0^{*}$ & Major groups 2 and 3 \\
\hline \multicolumn{3}{|l|}{ Paraguay } \\
\hline Pre-School and Elementary Teachers & $380-387^{*}$ & $233,331,332$ \\
\hline High School Teachers & $360-370^{*}$ & 232 \\
\hline Other Professionals and Technicians & Major group $0^{*}$ & Major groups 2 and 3 \\
\hline \multicolumn{3}{|l|}{ Peru } \\
\hline Pre-School and Elementary Teachers & $243,244^{\star \star \star \star *}$ & $243,244^{\star \star \star \star}$ \\
\hline High School Teachers & $242,246^{\star \star \star \star}$ & $242,246^{\star \star \star \star \star}$ \\
\hline Other Professionals and Technicians & Major groups 1 and $2^{\star \star \star}$ & Major groups 1 and $2^{\star}$ \\
\hline \multicolumn{3}{|l|}{ Uruguay } \\
\hline Pre-School and Elementary Teachers & $62,63^{\star}$ & $233,331,332$ \\
\hline High School Teachers & $61^{\star}$ & 232 \\
\hline Other Professionals and Technicians & Major group $0^{\star}$ & Major groups 2 and 3 \\
\hline
\end{tabular}

* Correspond to the occupational codes registered in MECOVI data bases

** Correspond to the "Composição dos Grupamentos Ocupacionais"

*** Correspond to the CELADE occupational codes of 1988

$* * * *$ Correspond to the INEC occupational codes of 1996

Note: all other occupational codes correspond to the ISCO-88 classification 
Table A2. Unexplained Earnings Gaps Controlling by the Full set of Observable Characteristics, by Country (by different measures of earnings)

\begin{tabular}{|c|c|c|c|c|c|}
\hline \multicolumn{6}{|c|}{ Pre-School and Elementary Teachers vis-à-vis Other Professionals and Technicians } \\
\hline \multirow{3}{*}{ Country } & \multicolumn{2}{|c|}{\begin{tabular}{|c|} 
Monthly earnings \\
\end{tabular}} & \multicolumn{3}{|c|}{\begin{tabular}{|c|} 
Yearly earnings \\
\end{tabular}} \\
\hline & Original gap & Full set & Original gap & \multicolumn{2}{|c|}{ Full set } \\
\hline & C-07 & C-07 & C-97 & C-97 & C-07 \\
\hline Bolivia & $194.4 \% 34.7 \%$ & $\begin{array}{|cc|}149.4 \% & 46.9 \% \\
(0.3) & (0.09) \\
\end{array}$ & $163.3 \% \quad 20.9 \%$ & $\begin{array}{c}123.2 \% \\
(0.27) \\
\end{array}$ & $\begin{array}{l}32.6 \% \\
(0.08) \\
\end{array}$ \\
\hline Brazil & $187.4 \% \quad 67.2 \%$ & $\begin{array}{|cc|}110.3 \% & 43.4 \% \\
(0.03) & (0.03) \\
\end{array}$ & $156.6 \% \quad 49.5 \%$ & $\begin{array}{l}87.4 \% \\
(0.03) \\
\end{array}$ & $\begin{array}{l}28.1 \% \\
(0.02) \\
\end{array}$ \\
\hline Chile & $77.4 \% \quad 51.3 \%$ & $\begin{array}{ll}63.1 \% & 23.2 \% \\
(0.07) & (0.06) \\
\end{array}$ & $59.9 \%$ & $\begin{array}{l}47.2 \% \\
(0.07) \\
\end{array}$ & $\begin{array}{l}11.3 \% \\
(0.05) \\
\end{array}$ \\
\hline Costa Rica & $10.5 \% \quad 15.9 \%$ & $\begin{array}{|cc|}-14.4 \% & 32.7 \% \\
(0.07) & (0.1) \\
\end{array}$ & $-0.7 \%$ & $\begin{array}{c}-23.2 \% \\
(0.06) \\
\end{array}$ & $\begin{array}{l}20.1 \% \\
(0.09) \\
\end{array}$ \\
\hline Dom. Rep. & $103.8 \% \quad 65.1 \%$ & $\begin{array}{ll}65.4 \% & 49.8 \% \\
(0.22) & (0.31) \\
\end{array}$ & $82.4 \%$ & $\begin{array}{c}46.8 \% \\
(0.2) \\
\end{array}$ & $\begin{array}{l}33.6 \% \\
(0.29) \\
\end{array}$ \\
\hline Ecuador & $46.0 \% \quad 74.2 \%$ & $\begin{array}{cc}79.5 \% & 31.7 \% \\
(0.46) & (0.1) \\
\end{array}$ & $56.7 \%$ & $\begin{array}{l}62.3 \% \\
(0.42) \\
\end{array}$ & $\begin{array}{c}20.3 \% \\
(0.1) \\
\end{array}$ \\
\hline El Salvador & $45.4 \% \quad 20.9 \%$ & $\begin{array}{cc}16.2 \% & 8.8 \% \\
(0.13) & (0.13) \\
\end{array}$ & $31.2 \%$ & $\begin{array}{c}4.4 \% \\
(0.12) \\
\end{array}$ & $\begin{array}{l}-2.0 \% \\
(0.12) \\
\end{array}$ \\
\hline Honduras & $23.8 \%$ & $\begin{array}{cc}13.5 \% & 28.7 \% \\
(0.08) & (0.1) \\
\end{array}$ & $12.1 \%$ & $\begin{array}{c}3.1 \% \\
(0.08) \\
\end{array}$ & $\begin{array}{l}15.9 \% \\
(0.09) \\
\end{array}$ \\
\hline Nicaragua & $189.9 \% \quad 157.0 \%$ & $\begin{array}{ll}88.0 \% & 85.5 \% \\
(0.24) & (0.21) \\
\end{array}$ & $161.5 \% 132.0 \%$ & $\begin{array}{l}71.0 \% \\
(0.21) \\
\end{array}$ & $\begin{array}{c}67.7 \% \\
(0.2) \\
\end{array}$ \\
\hline Panama & $69.9 \%$ & $\begin{array}{ll}34.2 \% & 35.4 \% \\
(0.12) & (0.08) \\
\end{array}$ & $54.0 \%$ & $\begin{array}{l}21.5 \% \\
(0.11) \\
\end{array}$ & $\begin{array}{l}22.2 \% \\
(0.07) \\
\end{array}$ \\
\hline Paraguay & $92.7 \%$ & $\begin{array}{ll}19.6 \% & 15.2 \% \\
(0.22) & (0.12) \\
\end{array}$ & $72.0 \%$ & $\begin{array}{l}6.9 \% \\
(0.2) \\
\end{array}$ & $\begin{array}{r}3.7 \% \\
(0.11) \\
\end{array}$ \\
\hline Peru & $106.2 \% \quad 82.5 \%$ & $\begin{array}{ll}58.9 \% & 49.5 \% \\
(0.35) & (0.09) \\
\end{array}$ & $64.3 \%$ & $\begin{array}{l}41.1 \% \\
(0.32) \\
\end{array}$ & $\begin{array}{l}34.1 \% \\
(0.08) \\
\end{array}$ \\
\hline Uruguay & $100.1 \% \quad 74.6 \%$ & $\begin{array}{cc}74.2 \% & 9.9 \% \\
(0.13) & (0.11) \\
\end{array}$ & $56.0 \%$ & $\begin{array}{l}54.4 \% \\
(0.12) \\
\end{array}$ & $\begin{array}{r}-2.1 \% \\
(0.1) \\
\end{array}$ \\
\hline $\begin{array}{l}\text { Latin America } \\
\text { (13 countries) }\end{array}$ & $145.0 \% \quad 64.7 \%$ & $\begin{array}{ll}96.1 \% & 58.8 \% \\
(0.03) & (0.02) \\
\end{array}$ & $119.3 \% \quad 47.5 \%$ & $\begin{array}{l}75.0 \% \\
(0.03) \\
\end{array}$ & $\begin{array}{l}42.0 \% \\
(0.02) \\
\end{array}$ \\
\hline
\end{tabular}




\begin{tabular}{|c|c|c|c|c|c|c|c|}
\hline \multicolumn{8}{|c|}{ High School Teachers vis-à-vis Other Professionals and Technicians } \\
\hline \multirow{3}{*}{ Country } & \multicolumn{3}{|c|}{ Monthly earnings } & \multicolumn{4}{|c|}{ Yearly earnings } \\
\hline & Original gap & \multicolumn{2}{|c|}{ Full set } & \multicolumn{2}{|c|}{ Original gap } & \multicolumn{2}{|c|}{ Full set } \\
\hline & C-07 & C-97 & C-07 & C-97 & C-07 & C-97 & C-07 \\
\hline Bolivia & $158.7 \% \quad 45.1 \%$ & $\begin{array}{l}91.7 \% \\
(0.34) \\
\end{array}$ & $\begin{array}{l}28.4 \% \\
(0.11) \\
\end{array}$ & $131.4 \%$ & $30.3 \%$ & $\begin{array}{l}70.1 \% \\
(0.31) \\
\end{array}$ & $\begin{array}{l}15.9 \% \\
(0.11) \\
\end{array}$ \\
\hline Brasil & $58.4 \% \quad 18.4 \%$ & $\begin{array}{l}42.9 \% \\
(0.09) \\
\end{array}$ & $\begin{array}{l}21.9 \% \\
(0.07) \\
\end{array}$ & $41.4 \%$ & $5.8 \%$ & $\begin{array}{l}27.5 \% \\
(0.08) \\
\end{array}$ & $\begin{array}{c}9.1 \% \\
(0.06) \\
\end{array}$ \\
\hline Chile & $53.8 \% \quad 11.4 \%$ & $\begin{array}{l}71.5 \% \\
(0.14) \\
\end{array}$ & $\begin{array}{c}13.7 \% \\
(0.1) \\
\end{array}$ & $38.6 \%$ & $0.3 \%$ & $\begin{array}{l}53.7 \% \\
(0.13) \\
\end{array}$ & $\begin{array}{c}2.7 \% \\
(0.09) \\
\end{array}$ \\
\hline Costa Rica & $6.4 \%$ & $\begin{array}{l}-6.0 \% \\
(0.27) \\
\end{array}$ & $\begin{array}{l}27.5 \% \\
(0.13) \\
\end{array}$ & $-3.0 \%$ & $-4.0 \%$ & $\begin{array}{c}-15.7 \% \\
(0.25) \\
\end{array}$ & $\begin{array}{l}14.7 \% \\
(0.12) \\
\end{array}$ \\
\hline Dom. Rep. & $31.3 \% \quad 24.1 \%$ & $\begin{array}{r}153.0^{\circ} \\
(0.93) \\
\end{array}$ & $\begin{array}{l}18.9 \% \\
(0.41) \\
\end{array}$ & $17.5 \%$ & $11.4 \%$ & $\begin{array}{c}112.0 \% \\
(0.78) \\
\end{array}$ & $\begin{array}{c}6.9 \% \\
(0.36) \\
\end{array}$ \\
\hline Ecuador & $35.5 \% \quad 39.8 \%$ & $\begin{array}{l}43.2 \% \\
(0.38) \\
\end{array}$ & $\begin{array}{l}50.2 \% \\
(0.19) \\
\end{array}$ & $23.2 \%$ & $25.7 \%$ & $\begin{array}{l}32.7 \% \\
(0.36) \\
\end{array}$ & $\begin{array}{l}34.7 \% \\
(0.17) \\
\end{array}$ \\
\hline El Salvador & $50.2 \% \quad 22.7 \%$ & $\begin{array}{r}1.7 \% \\
(0.27) \\
\end{array}$ & $\begin{array}{c}1.7 \% \\
(0.22) \\
\end{array}$ & $35.6 \%$ & $10.6 \%$ & $\begin{array}{l}-9.0 \% \\
(0.26) \\
\end{array}$ & $\begin{array}{c}-8.2 \% \\
(0.2) \\
\end{array}$ \\
\hline Honduras & $-4.5 \%$ & $\begin{array}{r}4.2 \% \\
(0.13) \\
\end{array}$ & $\begin{array}{l}22.6 \% \\
(0.16) \\
\end{array}$ & $-17.7 \%$ & $-14.2 \%$ & $\begin{array}{l}-4.7 \% \\
(0.12) \\
\end{array}$ & $\begin{array}{c}8.9 \% \\
(0.15) \\
\end{array}$ \\
\hline Nicaragua & $43.2 \% \quad 112.4 \%$ & $\begin{array}{c}-42.2 \% \\
(0) \\
\end{array}$ & $\begin{array}{c}127.6 \% \\
(0.73) \\
\end{array}$ & $29.1 \%$ & $91.7 \%$ & $\begin{array}{c}-44.0 \% \\
(0) \\
\end{array}$ & $\begin{array}{c}105.8 \% \\
(0.66) \\
\end{array}$ \\
\hline Panama & $22.4 \%$ & $\begin{array}{l}55.7 \% \\
(0.18) \\
\end{array}$ & $\begin{array}{l}32.2 \% \\
(0.09) \\
\end{array}$ & $10.9 \%$ & $-5.9 \%$ & $\begin{array}{l}41.3 \% \\
(0.17) \\
\end{array}$ & $\begin{array}{l}19.3 \% \\
(0.08) \\
\end{array}$ \\
\hline Paraguay & $40.7 \% \quad 54.5 \%$ & \begin{tabular}{|c|}
$-21.3 \%$ \\
$(0.27)$ \\
\end{tabular} & $\begin{array}{l}45.8 \% \\
(0.34) \\
\end{array}$ & $25.6 \%$ & $38.7 \%$ & $\begin{array}{c}-30.8 \% \\
(0.25) \\
\end{array}$ & $\begin{array}{l}29.4 \% \\
(0.29) \\
\end{array}$ \\
\hline Peru & $80.6 \%$ & $\begin{array}{l}49.5 \% \\
(0.34)\end{array}$ & $\begin{array}{l}63.0 \% \\
(0.15)\end{array}$ & $62.4 \%$ & $53.4 \%$ & $\begin{array}{l}32.3 \% \\
(0.31)\end{array}$ & $\begin{array}{l}46.6 \% \\
(0.14)\end{array}$ \\
\hline Uruguay & $84.2 \%$ & $\begin{array}{l}72.8 \% \\
(0.17) \\
\end{array}$ & $\begin{array}{c}9.0 \% \\
(0.12) \\
\end{array}$ & $64.1 \%$ & $32.9 \%$ & $\begin{array}{l}53.1 \% \\
(0.15) \\
\end{array}$ & $\begin{array}{l}-2.8 \% \\
(0.11) \\
\end{array}$ \\
\hline $\begin{array}{l}\text { Latin America } \\
\text { (13 countries) }\end{array}$ & $61.8 \%$ & $\begin{array}{l}66.4 \% \\
(0.08)\end{array}$ & $\begin{array}{l}47.9 \% \\
(0.06)\end{array}$ & $44.8 \%$ & $12.3 \%$ & $\begin{array}{l}47.1 \% \\
(0.07)\end{array}$ & $\begin{array}{l}32.4 \% \\
(0.05)\end{array}$ \\
\hline
\end{tabular}


Table A3. Unexplained Earnings Gaps after Controlling by the Full set of Observable Characteristics and Job Tenure, by country (7 countries with data on job tenure)

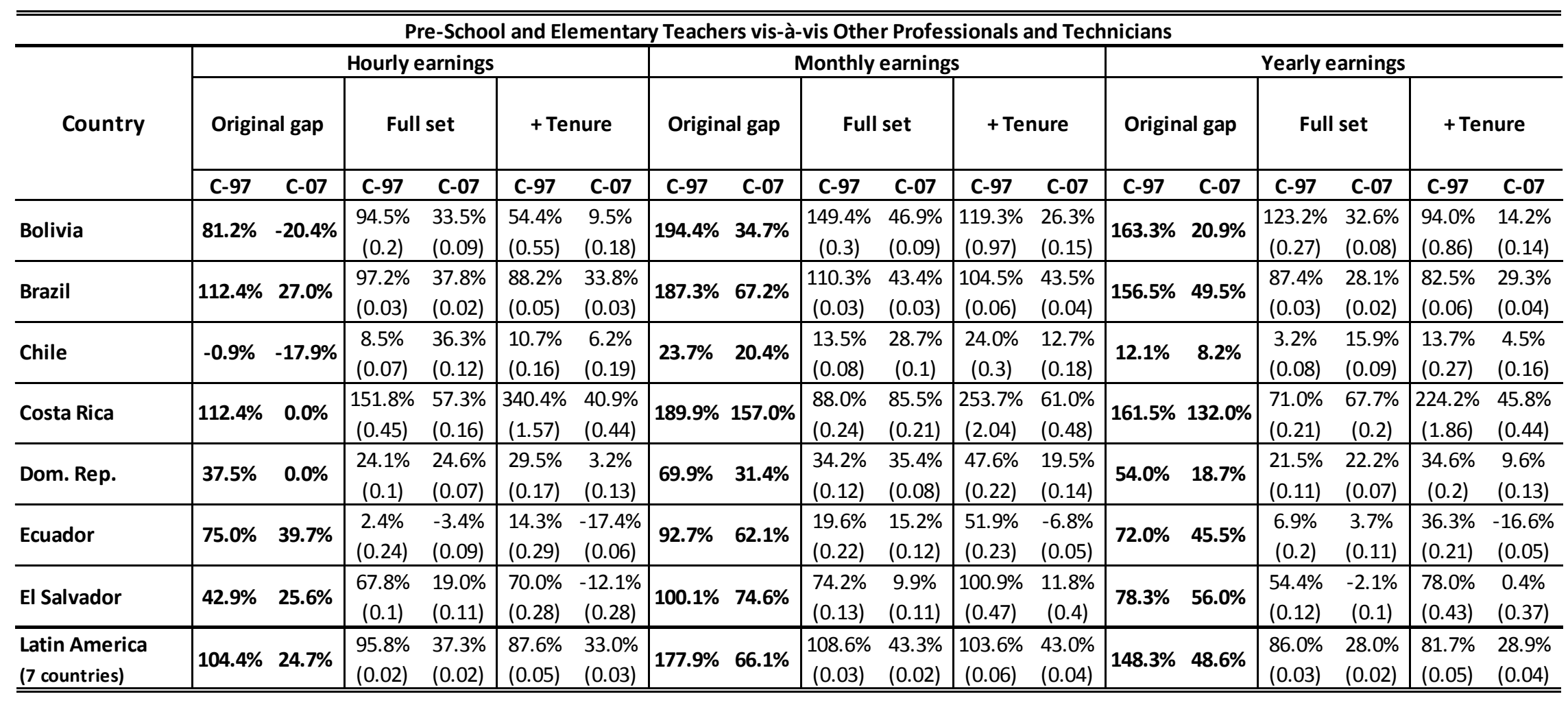




\begin{tabular}{|c|c|c|c|c|c|c|c|c|c|c|c|c|c|c|c|c|c|c|}
\hline \multicolumn{19}{|c|}{ High School Teachers vis-à-vis Other Professionals and Technicians } \\
\hline \multirow{3}{*}{ Country } & \multicolumn{6}{|c|}{ Hourly earnings } & \multicolumn{6}{|c|}{\begin{tabular}{|c|} 
Monthly earnings \\
\end{tabular}} & \multicolumn{6}{|c|}{ Yearly earnings } \\
\hline & \multicolumn{2}{|c|}{ Original gap } & \multicolumn{2}{|c|}{ Full set } & \multicolumn{2}{|c|}{+ Tenure } & \multicolumn{2}{|c|}{ Original gap } & \multicolumn{2}{|c|}{ Full set } & \multicolumn{2}{|c|}{+ Tenure } & \multicolumn{2}{|c|}{ Original gap } & \multicolumn{2}{|c|}{ Full set } & \multicolumn{2}{|c|}{ + Tenure } \\
\hline & C-97 & C-07 & C-97 & C-07 & C-97 & C-07 & C-97 & C-07 & C-97 & C-07 & C-97 & C-07 & C-97 & C-07 & C-97 & C-07 & C-97 & C-07 \\
\hline Bolivia & $35.1 \%$ & $-17.9 \%$ & $\begin{array}{l}61.4 \% \\
(0.24) \\
\end{array}$ & $\begin{array}{l}17.1 \% \\
(0.11) \\
\end{array}$ & $\begin{array}{c}174.0 \% \\
(0.52)\end{array}$ & $\begin{array}{c}-27.8 \% \\
(0.11) \\
\end{array}$ & $158.7 \%$ & $45.1 \%$ & $\begin{array}{l}91.7 \% \\
(0.34) \\
\end{array}$ & \begin{tabular}{l|}
$28.4 \%$ \\
$(0.11)$ \\
\end{tabular} & $\begin{array}{c}223.2 \% \\
(1.33)\end{array}$ & $\begin{array}{c}-28.4 \% \\
(0.16)\end{array}$ & $131.4 \%$ & $30.3 \%$ & $\begin{array}{l}70.1 \% \\
(0.31) \\
\end{array}$ & $\begin{array}{l}15.9 \% \\
(0.11) \\
\end{array}$ & $\begin{array}{c}195.0 \% \\
(1.21)\end{array}$ & $\begin{array}{c}-35.1 \% \\
(0.16) \\
\end{array}$ \\
\hline Brazil & $23.8 \%$ & $-3.2 \%$ & $\begin{array}{l}27.1 \% \\
(0.07)\end{array}$ & $\begin{array}{l}16.4 \% \\
(0.06)\end{array}$ & $\begin{array}{l}42.6 \% \\
(0.09)\end{array}$ & $\begin{array}{l}13.4 \% \\
(0.07)\end{array}$ & $58.3 \%$ & $18.4 \%$ & $\begin{array}{l}42.9 \% \\
(0.09)\end{array}$ & $\begin{array}{l}21.9 \% \\
(0.07)\end{array}$ & $\begin{array}{l}49.0 \% \\
(0.11)\end{array}$ & $\begin{array}{l}18.7 \% \\
(0.09)\end{array}$ & $41.3 \%$ & $5.8 \%$ & $\begin{array}{l}27.5 \% \\
(0.08)\end{array}$ & $\begin{array}{c}9.1 \% \\
(0.06)\end{array}$ & $\begin{array}{c}34.9 \% \\
(0.1)\end{array}$ & $\begin{array}{c}6.9 \% \\
(0.08)\end{array}$ \\
\hline Chile & $-22.2 \%$ & $-19.0 \%$ & $\begin{array}{l}-2.4 \% \\
(0.09) \\
\end{array}$ & \begin{tabular}{l|}
$12.0 \%$ \\
$(0.13)$ \\
\end{tabular} & $\begin{array}{c}36.5 \% \\
(0) \\
\end{array}$ & $\begin{array}{l}-8.8 \% \\
(0.25) \\
\end{array}$ & $-9.2 \%$ & $-4.5 \%$ & $\begin{array}{c}4.2 \% \\
(0.13) \\
\end{array}$ & $\begin{array}{l}22.6 \% \\
(0.16) \\
\end{array}$ & $\begin{array}{c}66.6 \% \\
(0) \\
\end{array}$ & $\begin{array}{c}19.6 \% \\
(0.2) \\
\end{array}$ & $-17.7 \%$ & $-14.2 \%$ & $\begin{array}{l}-4.7 \% \\
(0.12) \\
\end{array}$ & $\begin{array}{c}8.9 \% \\
(0.15) \\
\end{array}$ & $\begin{array}{c}55.0 \% \\
(0) \\
\end{array}$ & $\begin{array}{l}11.8 \% \\
(0.18) \\
\end{array}$ \\
\hline Costa Rica & $28.9 \%$ & $60.9 \%$ & $\begin{array}{c}28.2 \% \\
(0)\end{array}$ & $\begin{array}{l}51.5 \% \\
(0.42)\end{array}$ & $\begin{array}{c}0.0 \% \\
(0)\end{array}$ & $\begin{array}{c}-26.0 \% \\
(0.75)\end{array}$ & $43.2 \%$ & $112.4 \%$ & $\begin{array}{c}-42.2 \% \\
(0)\end{array}$ & $\begin{array}{c}127.6 \% \\
(0.73)\end{array}$ & $\begin{array}{c}0.0 \% \\
(0)\end{array}$ & $\begin{array}{c}-25.1 \% \\
(0.77)\end{array}$ & $29.1 \%$ & $91.7 \%$ & $\begin{array}{c}-44.0 \% \\
(0)\end{array}$ & $\begin{array}{c}105.8 \% \\
(0.66)\end{array}$ & $\begin{array}{c}0.0 \% \\
(0)\end{array}$ & $\begin{array}{c}-35.4 \% \\
(0.71)\end{array}$ \\
\hline Dom. Rep. & $-0.6 \%$ & $-3.7 \%$ & $\begin{array}{l}37.9 \% \\
(0.13) \\
\end{array}$ & $\begin{array}{l}21.5 \% \\
(0.08) \\
\end{array}$ & $\begin{array}{c}9.0 \% \\
(0.24) \\
\end{array}$ & \begin{tabular}{l|}
$14.1 \%$ \\
$(0.19)$ \\
\end{tabular} & $22.4 \%$ & $4.2 \%$ & $\begin{array}{l}55.7 \% \\
(0.18) \\
\end{array}$ & $\begin{array}{l}32.2 \% \\
(0.09) \\
\end{array}$ & $\begin{array}{l}28.6 \% \\
(0.36) \\
\end{array}$ & $\begin{array}{l}17.2 \% \\
(0.23) \\
\end{array}$ & $10.9 \%$ & $-5.9 \%$ & $\begin{array}{l}41.3 \% \\
(0.17) \\
\end{array}$ & $\begin{array}{l}19.3 \% \\
(0.08) \\
\end{array}$ & $\begin{array}{l}17.1 \% \\
(0.33) \\
\end{array}$ & $\begin{array}{c}7.8 \% \\
(0.21) \\
\end{array}$ \\
\hline Ecuador & $6.5 \%$ & $10.9 \%$ & $\begin{array}{c}-21.1 \% \\
(0.03)\end{array}$ & $\begin{array}{l}40.9 \% \\
(0.23) \\
\end{array}$ & $\begin{array}{c}0.0 \% \\
(0)\end{array}$ & $\begin{array}{c}20.3 \% \\
(0)\end{array}$ & $40.7 \%$ & $54.8 \%$ & $\begin{array}{c}-21.3 \% \\
(0.27) \\
\end{array}$ & $\begin{array}{l}45.8 \% \\
(0.34) \\
\end{array}$ & $\begin{array}{c}0.0 \% \\
(0)\end{array}$ & $\begin{array}{c}8.1 \% \\
(0)\end{array}$ & $25.6 \%$ & $38.9 \%$ & $\begin{array}{c}-30.8 \% \\
(0.25) \\
\end{array}$ & $\begin{array}{l}29.4 \% \\
(0.29) \\
\end{array}$ & $\begin{array}{c}0.0 \% \\
(0)\end{array}$ & $\begin{array}{c}1.5 \% \\
(0)\end{array}$ \\
\hline El Salvador & $-0.9 \%$ & $-0.9 \%$ & $\begin{array}{l}62.5 \% \\
(0.13) \\
\end{array}$ & $\begin{array}{l}12.5 \% \\
(0.11) \\
\end{array}$ & $\begin{array}{c}30.9 \% \\
(0.3) \\
\end{array}$ & \begin{tabular}{c|}
$130.5 \%$ \\
$(0.47)$ \\
\end{tabular} & $-0.9 \%$ & $-0.9 \%$ & $\begin{array}{l}72.8 \% \\
(0.17) \\
\end{array}$ & $\begin{array}{c}9.0 \% \\
(0.12) \\
\end{array}$ & $\begin{array}{c}6.8 \% \\
(0.38) \\
\end{array}$ & $\begin{array}{c}141.7 \% \\
(0.44) \\
\end{array}$ & $-0.9 \%$ & $-0.9 \%$ & $\begin{array}{l}53.1 \% \\
(0.15) \\
\end{array}$ & $\begin{array}{l}-2.8 \% \\
(0.11) \\
\end{array}$ & $\begin{array}{l}-7.0 \% \\
(0.36) \\
\end{array}$ & $\begin{array}{c}126.4 \% \\
(0.4) \\
\end{array}$ \\
\hline $\begin{array}{l}\text { Latin America } \\
\text { ( } 7 \text { countries) }\end{array}$ & $25.6 \%$ & $-2.7 \%$ & $\begin{array}{l}29.1 \% \\
(0.06)\end{array}$ & $\begin{array}{l}16.7 \% \\
(0.06)\end{array}$ & $\begin{array}{l}45.6 \% \\
(0.09) \\
\end{array}$ & $\begin{array}{l}13.0 \% \\
(0.07) \\
\end{array}$ & $64.3 \%$ & $20.9 \%$ & $\begin{array}{l}45.7 \% \\
(0.08)\end{array}$ & $\begin{array}{l}22.8 \% \\
(0.07) \\
\end{array}$ & $\begin{array}{l}53.2 \% \\
(0.11) \\
\end{array}$ & $\begin{array}{l}18.7 \% \\
(0.09) \\
\end{array}$ & $46.7 \%$ & $8.1 \%$ & $\begin{array}{l}29.7 \% \\
(0.07) \\
\end{array}$ & $\begin{array}{c}9.8 \% \\
(0.06) \\
\end{array}$ & $\begin{array}{c}38.2 \% \\
(0.1) \\
\end{array}$ & $\begin{array}{c}6.8 \% \\
(0.08) \\
\end{array}$ \\
\hline
\end{tabular}

Source: Authors' calculations based on household surveys.

Standard errors in parentheses 
Figure A1. Confidence Intervals for the Unexplained Earnings Gap by Different Characteristics for PreSchool and Elementary School Teachers versus Other Professionals and Technicians

(after controlling by the full set of characteristics)
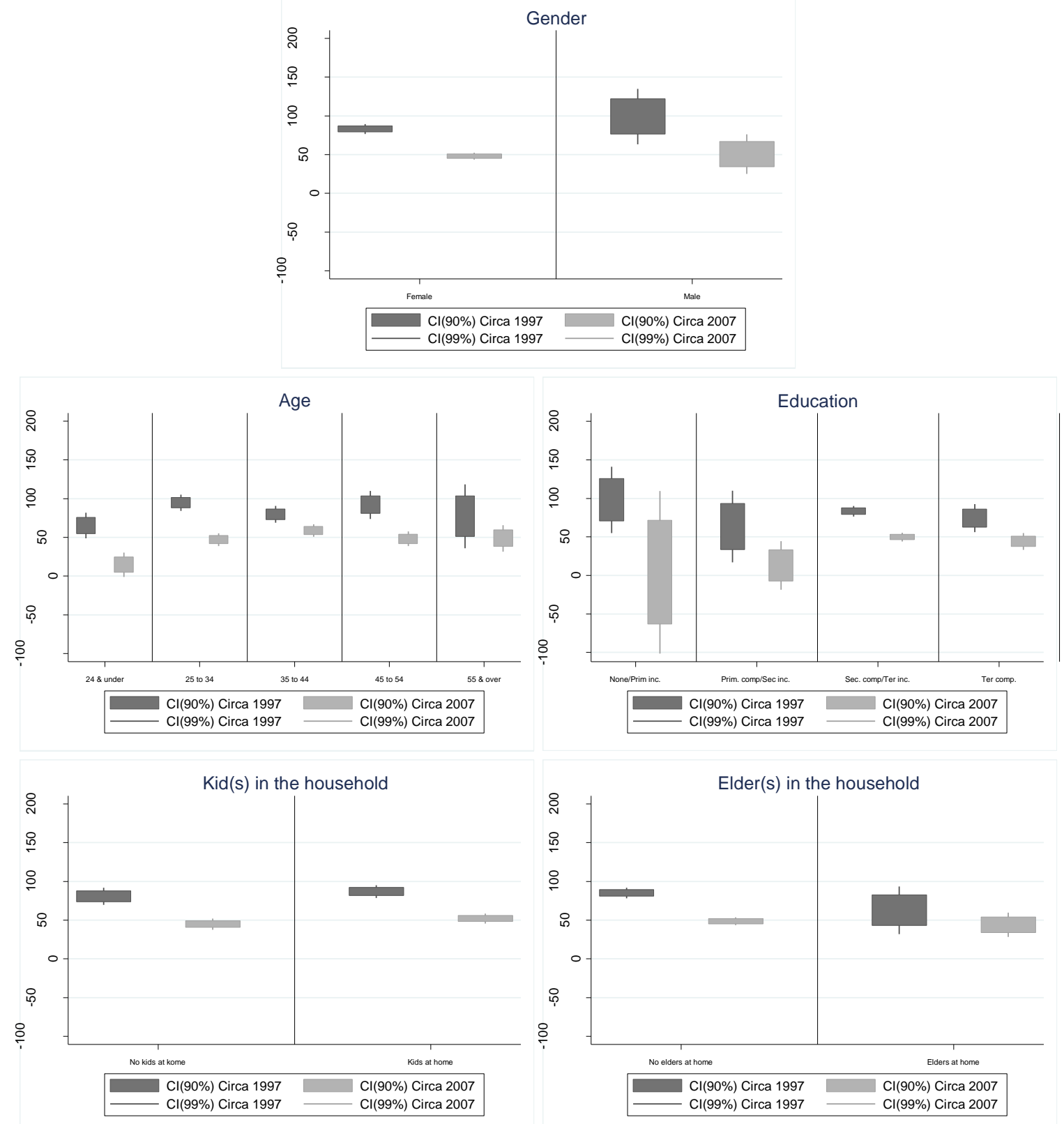

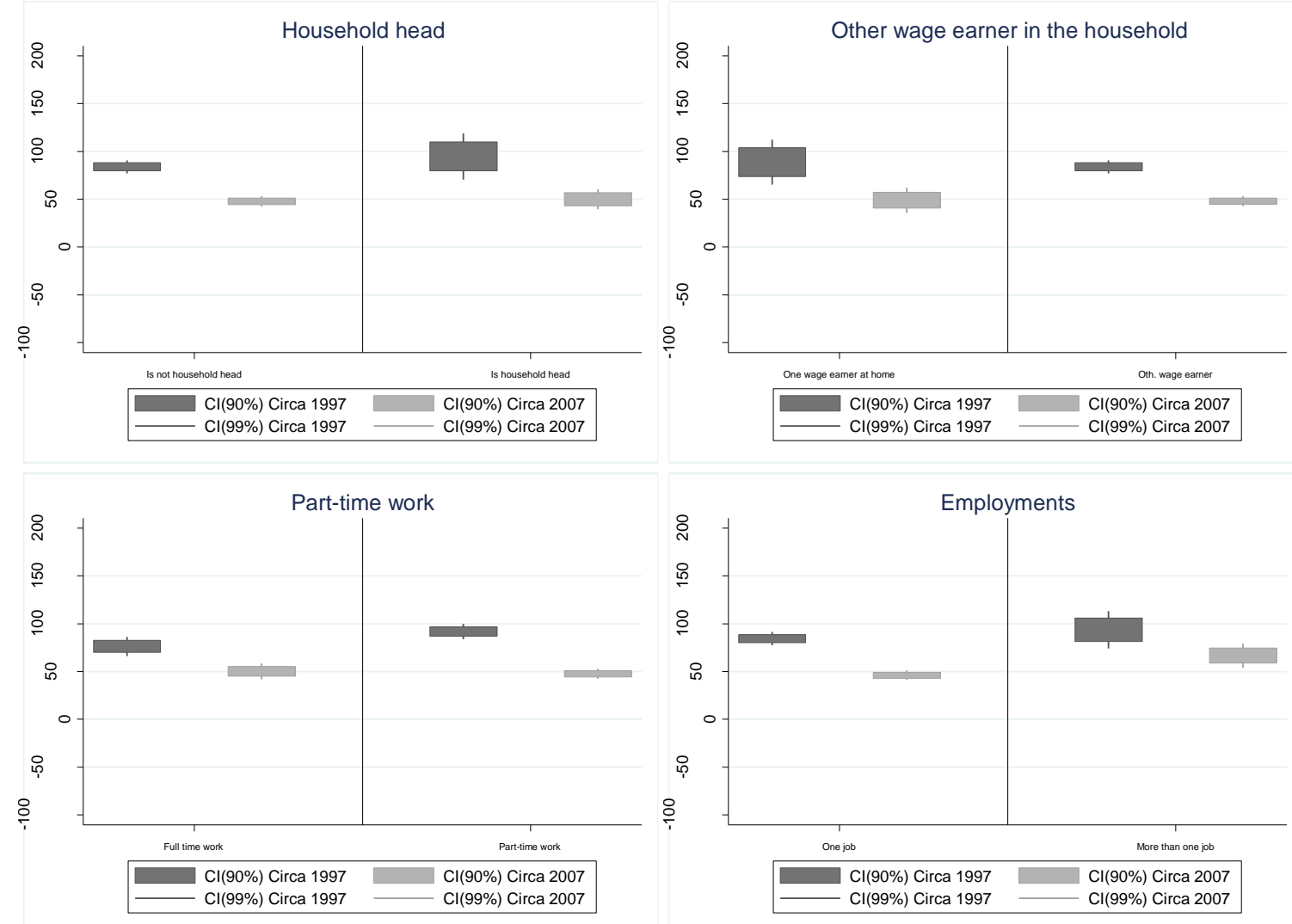

Source: Authors' calculations based on household surveys

Note: Boxes show 90 percent confidence intervals for unexplained earnings; whiskers show 99 percent confidence intervals.

Figure A2. Confidence Intervals for the Unexplained Earnings Gap by Different Characteristics for High School Teachers versus Other Professionals and Technicians (after controlling by the full set of characteristics)

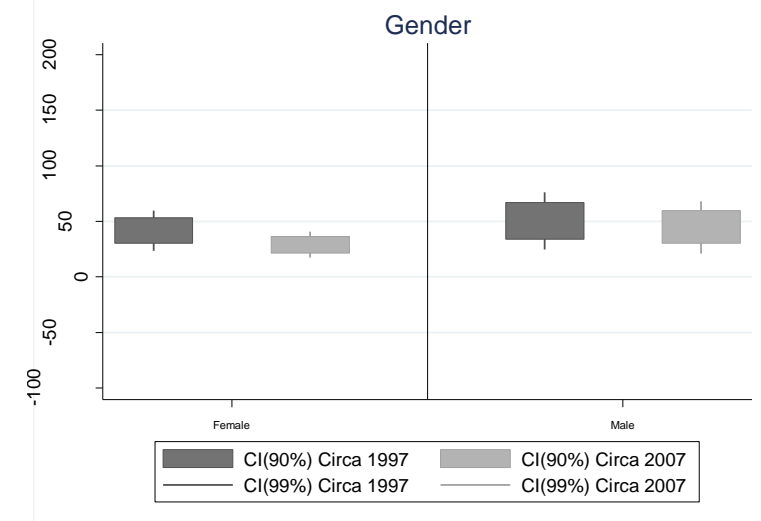



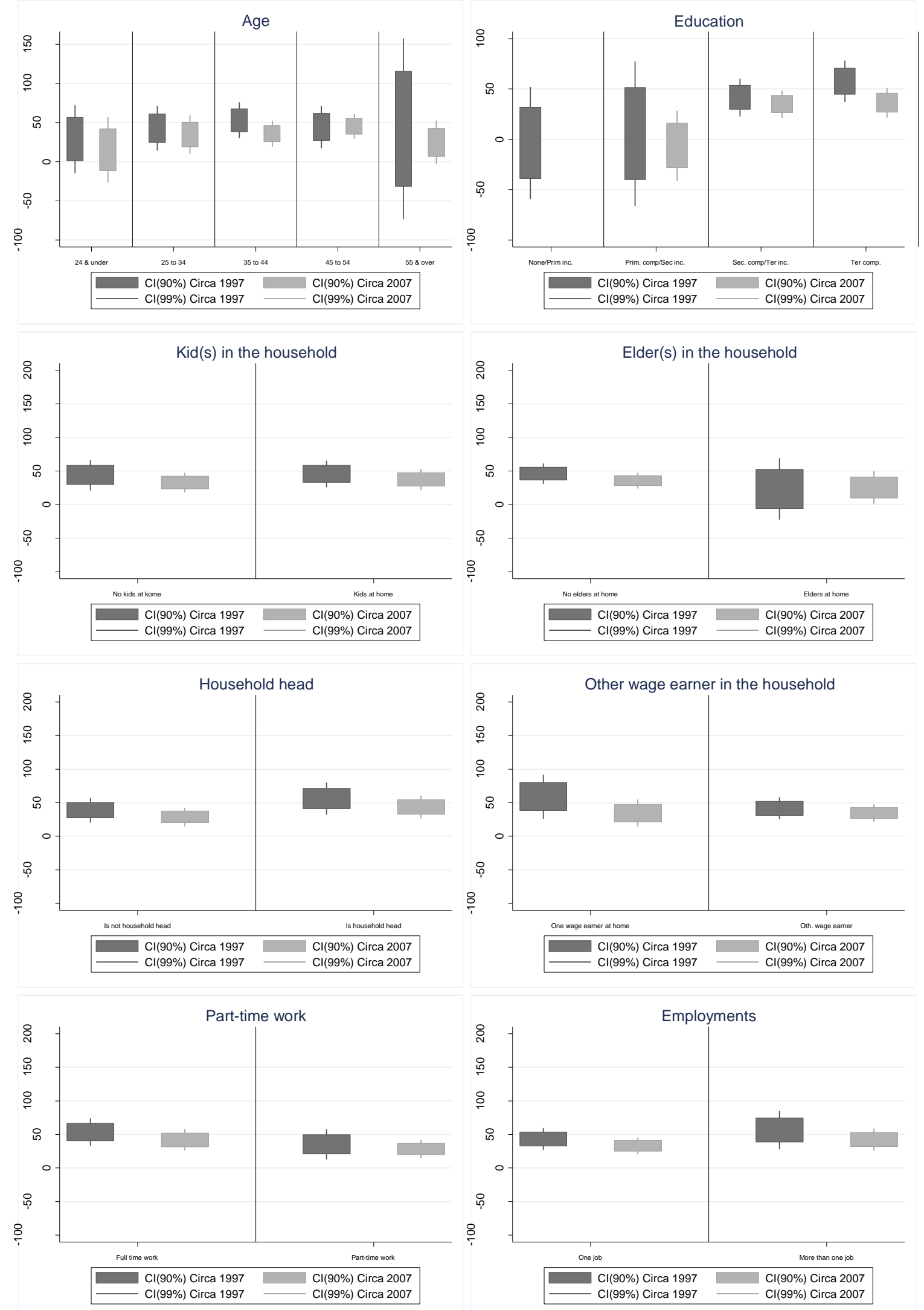

Source: Authors' calculations based on household surveys 